\title{
Research Paper \\ Efficacy of Cognitive-Behavioral Hypnotherapy on Body Dysmorphic Disorder: Case Study
}

\author{
Morteza Abbarin ${ }^{1},{ }^{*}$ Mehdi Zemestani², Mehdi Rabiei ${ }^{3}$, Amir Houshang Bagheri $^{4}$
}

\begin{abstract}
1. MSc. in Clinical Psychology, Department of Psychology, Faculty of Literature and Social Sciences, University of Kurdistan, Sanandaj, Iran. 2. PhD in Psychology, Assistant Professor, Department of Psychology, Faculty of Humanities and Social Sciences, University of Kurdistan, Sanandaj, Iran. 3. PhD in Psychology, Assistant Professor, Department of Psychology, Faculty of Medicine, Baqiyatallah University of Medical Sciences, Tehran, Iran.
\end{abstract} 4. Psychiatrist, Branch of Hypnotherapy, Psychiatric Committee, Iranian Psychiatric Association, Tehran, Iran.

Received: 21 Jun. 2016

Accepted: 15 Apr. 2017

Key words: Cognitive behavioral hypnotherapy, Body Dysmorphic Disorder (BDD), Depression, Disability
Cftation: Abbarin M, Zemestani M, Rabiei M, Bagheri AH. [Efficacy of Cognitive-Behavioral Hypnotherapy on Body Dysmorphic Disorder: Case Study (Persian)]. Iranian Journal of Psychiatry and Clinical Psychology. 2018; 23(4):394407. https://doi.org/10.29252/nirp.ijpcp.23.4.394

https://doi.org/10.29252/nirp.ijpcp.23.4.394

\section{A B S TRACT}

Objectives This study examined the effect of cognitive behavioral hypnotherapy on Body Dysmorphic Disorder (BDD), depression, and disability of patients.

Methods In this article, we present a single subject experimental design with multiple baselines. The subjects of the study were patients with BDD in Sanandaj city. Purposive sampling was used, and after obtaining diagnostic interview and qualifications of the study, subjects underwent the treatment process. The Yale-Brown Obsessive Compulsive Scale modified for Body Dysmorphic Disorder (BDD-YBOCS), Beck Depression Inventory (DBI-II), Sheehan Disability Scale (SDS), Millon Clinical Multiaxial Inventory-III (MCMI-III) and clinical interview were used as diagnostic tools. Cognitive-behavioral hypnotherapy was employed for 15 sessions of one and a half hours and for two sessions per week. For data analysis, we used a single-case analysis using graphs and comparison of mean and median positions.

Results The results showed that participants' scores in BDD, depression, and disability decreased noticeably. Treatment outcomes were continued up to four months of follow-up period as well.

Conclusion Cognitive-behavioral hypnotherapy is effective in improving BDD, depression, and disability of these patients.

\section{Extended Abstract}

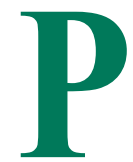

\section{Introduction}

atients with Body Dysmorphic Disorder (BDD) are preoccupied with imaginary defects in their appearances which bring about significant problems in important functioning areas [1].

According to the cognitive model of BDD, the progression of dysfunctional beliefs leads to rituals, avoidant behaviors, and negative thoughts. Negative emotions (e.g. fear, anxiety, and shame) activated by thoughts and negative beliefs, encourage efforts to neutralize these emotions through avoidant behavior and rituals. These rituals and avoidant behaviors can alleviate painful emotions temporally, but they are negatively reinforced and actually maintain the dysfunctional BDD beliefs and behaviors [3].

In clinical practice, patients with BDD are usually considered as resistant to treatment, and many fail to respond or even make a complete recovery [9]. Cognitive behavioral model of BDD emphasizes the role of imagination on maintenance of disorder [10-12]. Cognitive behavioral

.

* Corresponding Author:

Mehdi Zemestani, PhD

Address: Department of Psychology, Faculty of Literature and Social Sciences, University of Kurdistan, Sanandaj, Iran.

Tel: +98 (912) 4374452

E-mail: m.zemestani@uok.ac.ir 
hypnotherapy assumes that the causes of most psychological disturbances are negative forms of self-hypnosis, in which negative thoughts are accepted without being criticized and even without conscious knowledge [13]. Hypnotherapy as an effective and complementary therapy is used for body image related disorders (eating disorders) [24, 25]. To the best of our knowledge, no research on the type of the effectiveness of interventions based on hypnosis has been performed on BBD.

The body image has mental, sensory, and body dimensions and studies support the increasing effectiveness of cognitive behavioral therapy in conjunction with hypnosis [27]. Hence, the present study was undertaken to determine the efficacy of cognitive behavioral hypnotherapy on BBD, depression, and disability of these patients.

\section{Methods}

In this article, we present a single subject experimental design with three baselines. The study population was patients with BBD in Sanandaj city. Purposive sampling was used, and after obtaining diagnostic interview and qualifications of the study, subjects underwent the treatment process. Two male and one female patient who were diagnosed with BBD and satisfied the inclusion criteria took part in the study.
The Yale-Brown Obsessive Compulsive Scale modified for BBD (BDD-YBOCS), Beck Depression Inventory (DBI-II), Sheehan Disability Scale (SDS), Millon Clinical Multiaxial Inventory-III (MCMI-III), and clinical interview were used every week in baseline phase and twice a week in treatment condition at the beginning of each session. Cognitive-behavioral hypnotherapy was employed for 15 sessions of one and a half hours and for two sessions per week.

We used Wilhelm and colleagues' manual of cognitive behavioral therapy for BBD [7] and Alladin's cognitive hypnotherapeutic techniques [26] to develope the protocol for this study. This protocol was acknowledged by two specialist in hypnotherapy as well as the treatment BBD (third and fourth authors). For analysis of data, we used a single-case analysis using graphs and comparison of mean and median positions. The study was registered in Iranian Registry of Clinical Trials (IRCT) having code IRCT2016050425838N2.

The first patient was a 30-year-old man. The main concern was the weak bones, hairs, and jaws, but he was pretty much preoccupied with all parts of his body. The second patient was a 31-year-old woman. Her main concern was her hair, and her way of walking and standing. The third patient was a 21-year-old man. His main concern was the nose, the sound, the parenthesis of the foot, and the hair.

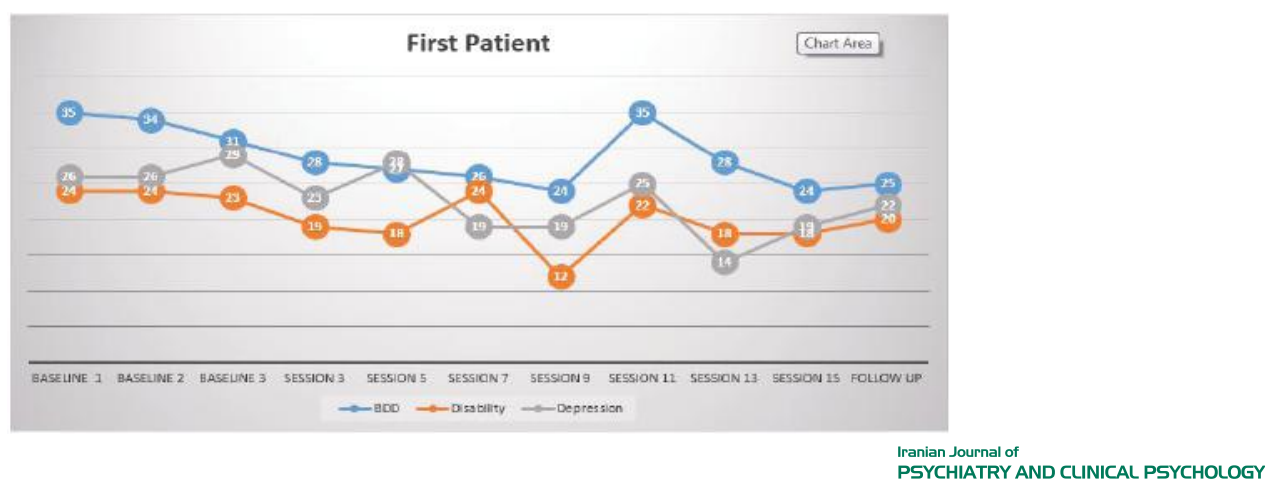

Figure 1. Effectiveness of cognitive behavioral hypnotherapy on $\mathrm{BBD}$, depression, and disability of the first patient

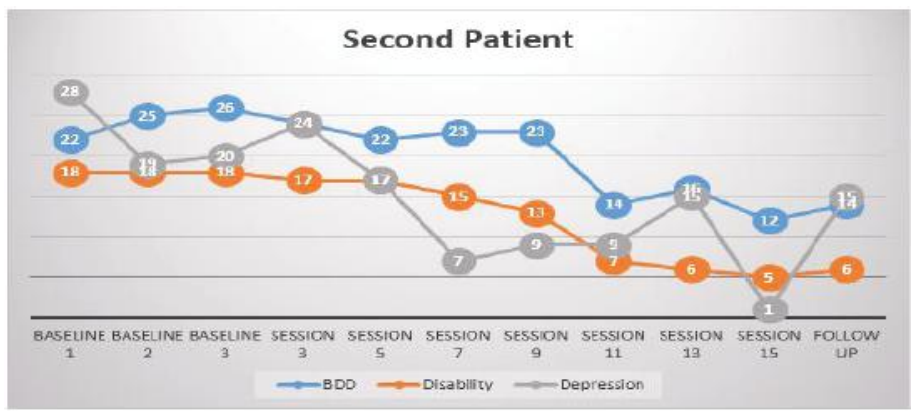

Iranian Journal of
PSYCHIATRY AND CUINICAL PSYCHOLOGY

Figure 2. Effectiveness of cognitive behavioral hypnotherapy on $\mathrm{BBD}$, depression and disability of the second patient 


\section{Results}

The results showed that the participants' scores in BBD, depression, and disability decreased markedly. Two out of three patients (patient number 2 and 3) achieved the response to treatment criteria ( $\geq 30 \%$ reduction in BDDYBOCS score from baseline) after treatment (50\% and $37 \%$ reduction) and after four months follow-up ( $44 \%$ and $41 \%$ reduction) period. Patient number one was diagnosed with comorbid OCD and OCPD, and these may affect his treatment outcome (28\% reduction) (Figure 1). In addition to improving the scores of BDD, the scores of disability and depression improved in all three patients.

Participants' scores in depression and disability significantly decreased in all patients after treatment $33 \%$, $95 \%$, and $89 \%$ reduction in depression scores and $22 \%$, $72 \%$, and $73 \%$ reduction in disability scores for patients 1,2 , and 3 , respectively) and at follow-up period ( $18 \%$, $33 \%$, and $37 \%$ reduction in depression scores and $16 \%$, $66 \%$, and $78 \%$ reduction in disability scores for patients 1,2 , and 3 , respectively). Figures 1 and 2 show a decrease in the scores in the treatment phase relative to the baseline period. The decrease in the follow-up period has also been relatively stable, and the level of change in the mean level is also evident.

\section{Discussion}

The present study reports the development and extension of the hypnotherapy supplementary protocol to cognitivebehavioral interventions for BBD. The results show proper efficacy in improving BBD, depression, and disability of these patients. Considering the benefits of hypnotherapy to improve BBD in patients as suggested in this study, it seems reasonable to incorporate hypnosis in cognitive-behavioral therapy for BBD.

The low number of participants and the compression of treatment protocol can be among the factors influencing the findings and the limitations of this research. Given the preliminary nature of this study and its single subject design, there is a need for further investigation in the future before generalizing the results. Moreover, the effect of variables such as hypnotizability of patients and comorbidities on the effectiveness of treatment should be considered for future investigations.

\section{Ethical Considerations}

Compliance With Ethical Guideline
Research was carried out with written informed consent, confidentiality of data, and other important points of research ethics.

\section{Funding}

This research was extracted from the MSc. thesis of the first author in the Department of Psychology, Faculty of Literature and Social Sciences, University of Kurdistan, Sanandaj, Iran.

\section{Conflict of Interest}

The authors declare no conflict of interest.

\section{Acknowledgements}

The authors of the present article are grateful to all the participants in the research and all those who have facilitated the implementation of the research and facilitated their implementation. 


\title{
كار ايي هيينوترايى شناختى رفتارى بر اختلال بدشكلى بدن: مطالعه موردى \\ مرتضى آبرين'، "مهدى زمستانى"، مهدى ربيعى"، اميرهوشنى باقرى"
}

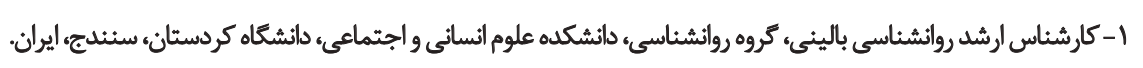

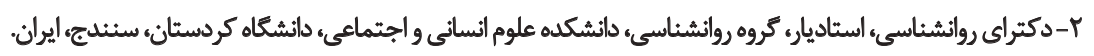

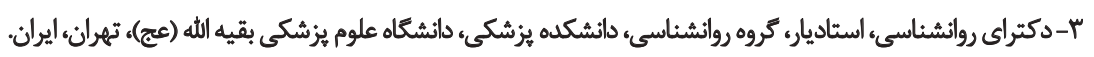

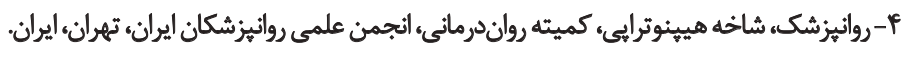

\begin{abstract}
حكيد

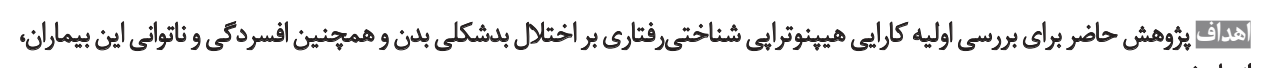

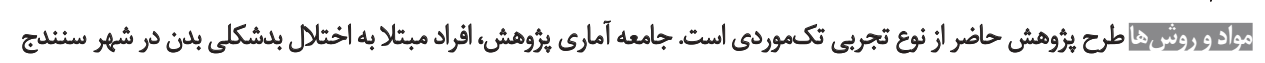

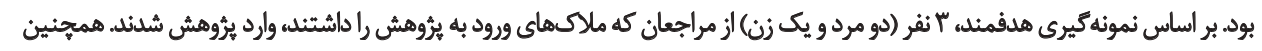

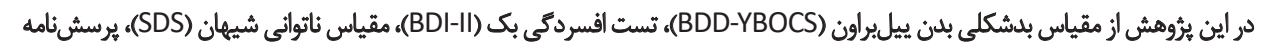

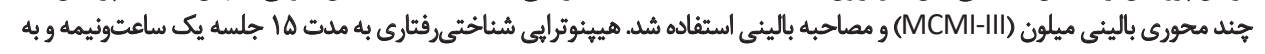

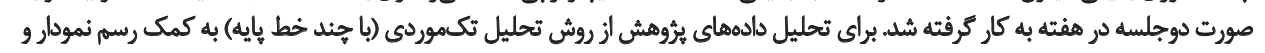

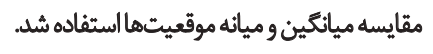

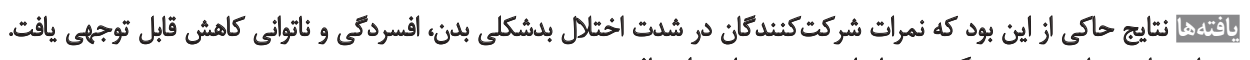

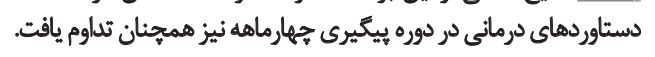

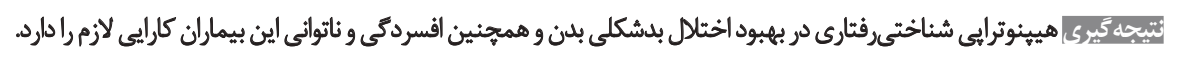

تاريخ دريافت: الثير

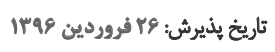

كليدوازئها:

هيينوترائى شناختى فئتاري،

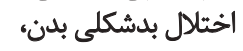
افسردكى، ناتواني

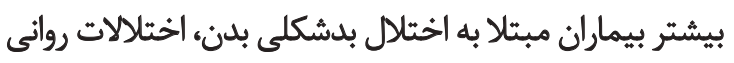

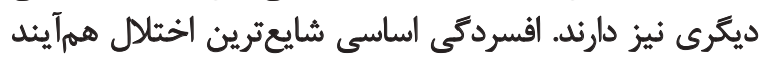

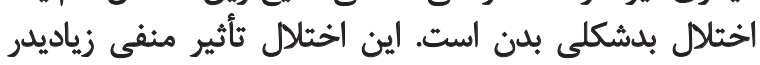
كيفيت زندكى دارد [ب].

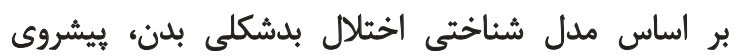

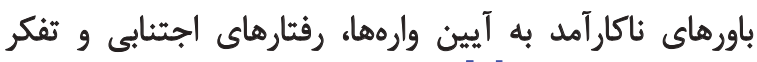

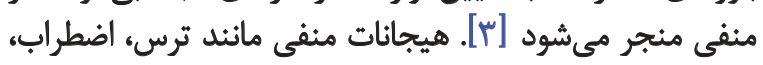

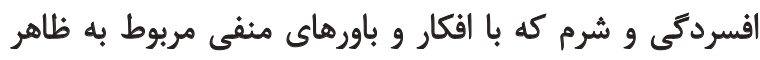

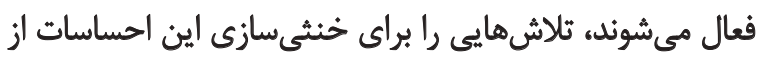

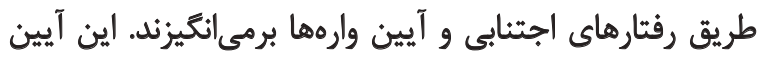

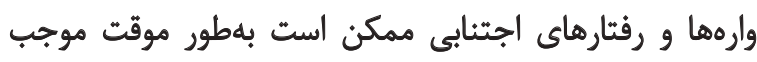

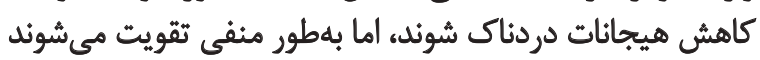

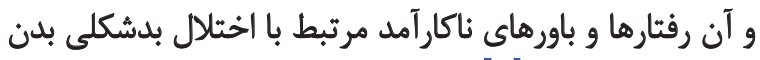

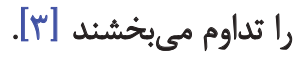

مقدمه

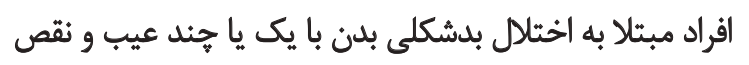

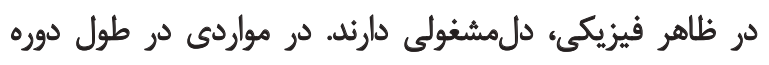

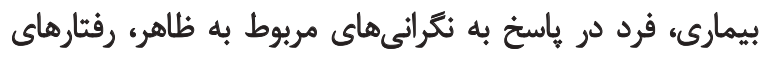

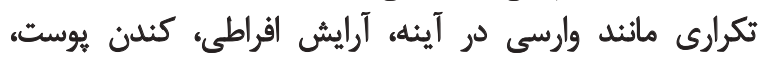

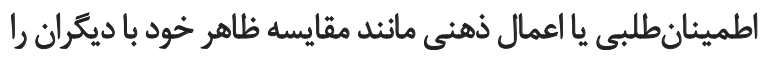

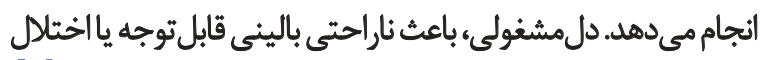

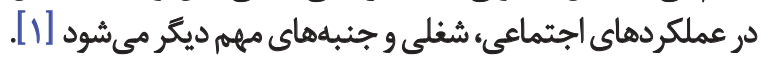

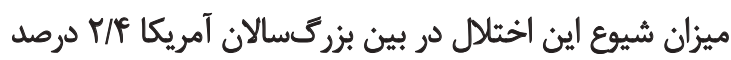

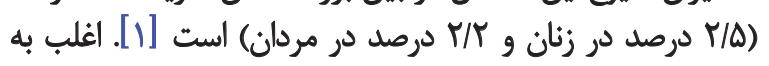

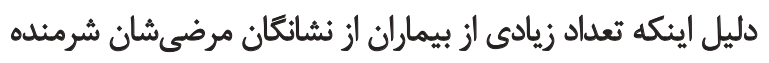

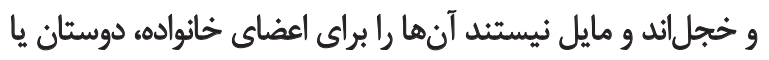

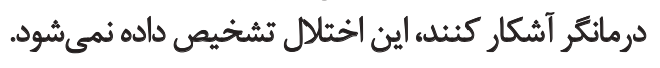


بازسازى شناختى در هيينوتيزم"، اصلاح و وپاككردن يرونده

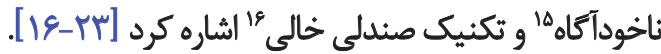
اخرجه اثربخشى مداخلات مبتنى بر هيينوتيزم در درمان

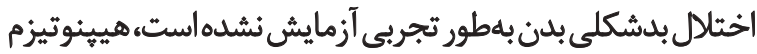

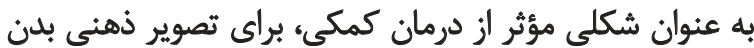

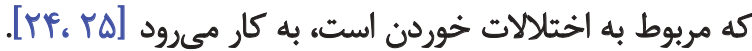

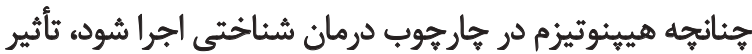

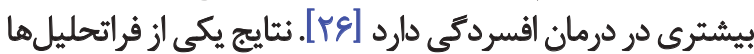

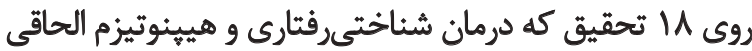

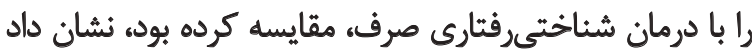

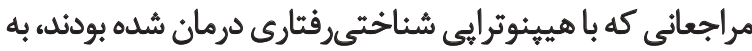

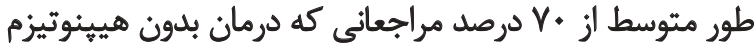

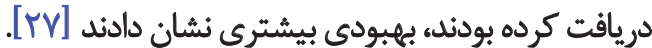

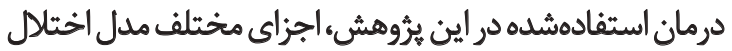

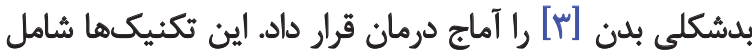

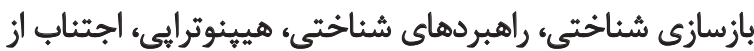

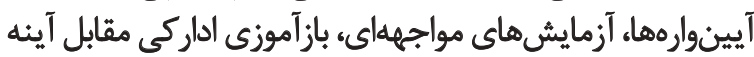

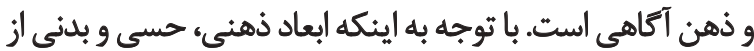

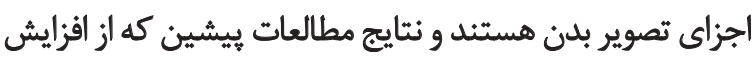

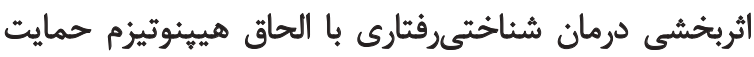

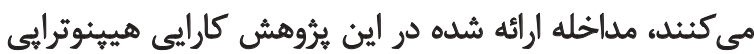

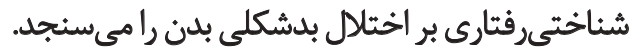

كرأزوهش حاضركه با كد IRCT2016050425838N2

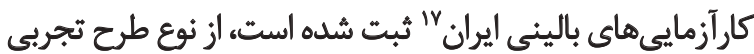

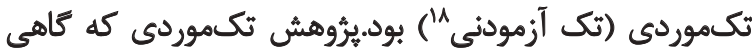

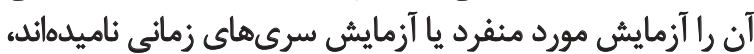

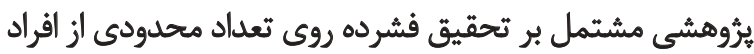

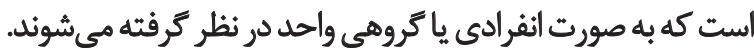

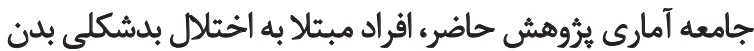

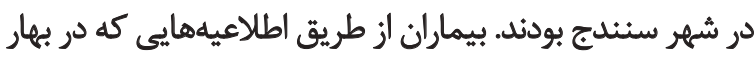

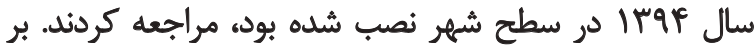

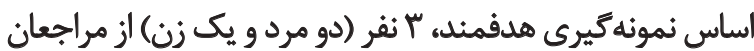

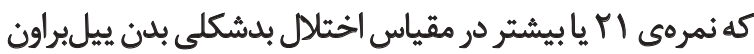

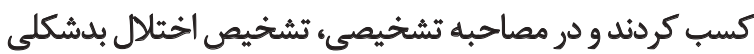

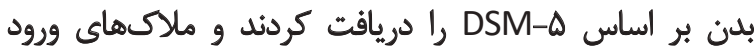

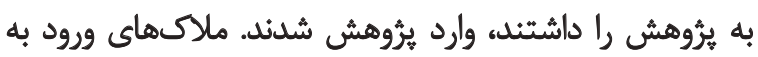

14. Cognitive restructuring under hypnosis

15. Editing and deleting the "unconscious file"

16. Empty-chair technique

17. Iranian Registry of Clinical Trials (IRCT)

18. Single subject
با وجود شيوع اختّلال بدشكلى بلدن، تحقيقات درمانى درباره اين

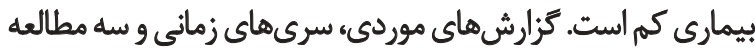

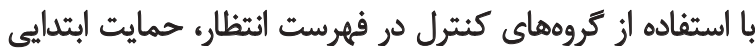

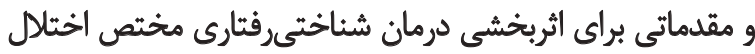

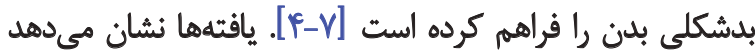

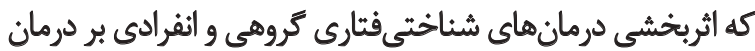

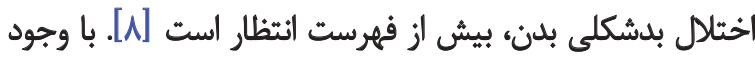

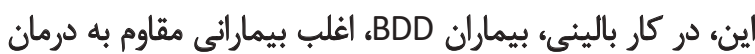

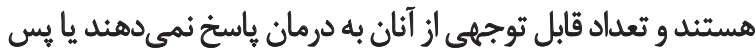

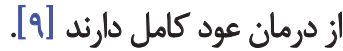

مدل شناختىرفتارى BDD'، بر نقش تصويرسازى ذهنى در

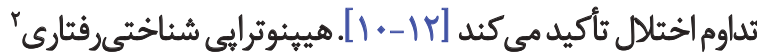

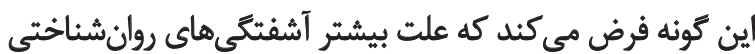

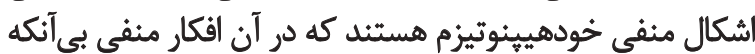

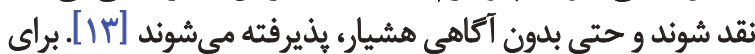

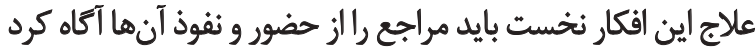

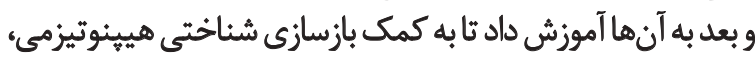

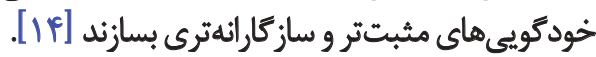

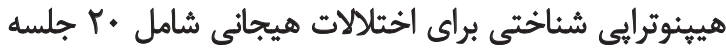

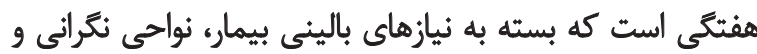

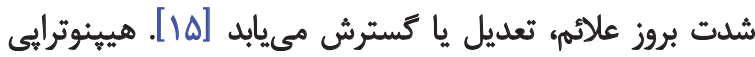

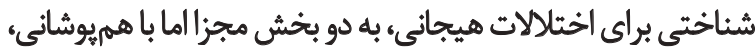

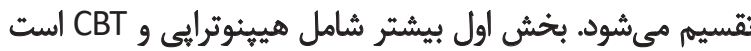

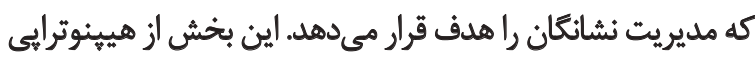

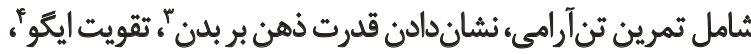

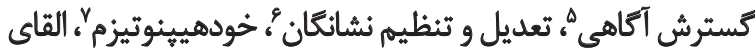

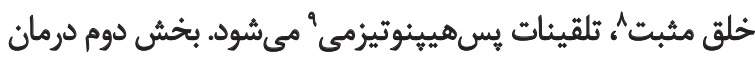

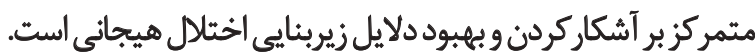

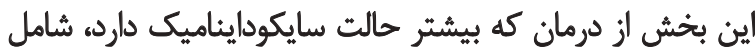

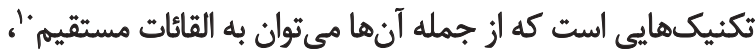

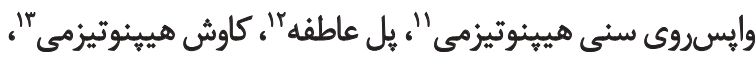

1. Body Dysmorphic Disorder

2. Cognitive-behavioral hypnotherapy

3. Demonstration of the power of mind over the body

4. Ego-strengthening

5. Expansion of awareness

6. Modulation and regulation of symptoms

7. Self-hypnosis

8. Positive mood induction

9. Posthypnotic suggestions

10. Direct suggestions

11. Hypnotic age regression

12. Affect bridge

13. Hypnotic exploration 
بدشكلى بلدن احير دارند. ضريب آلفاي كرونباخ در اين يُروهش

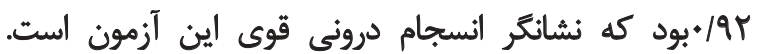

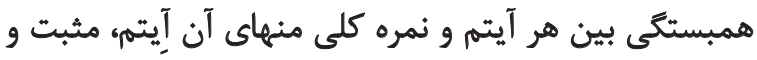

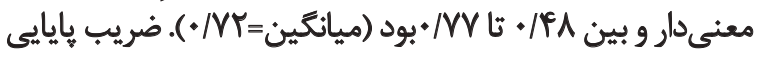

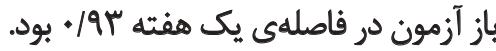

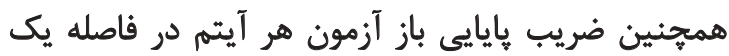

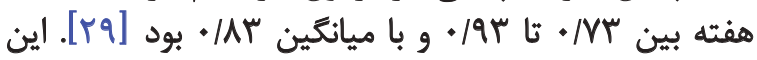

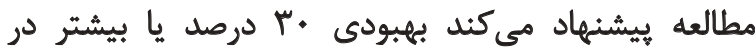

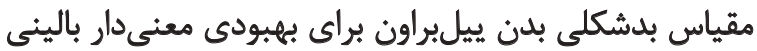

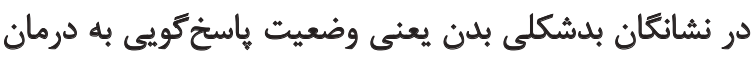

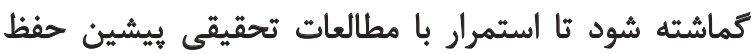

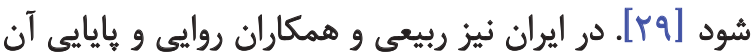

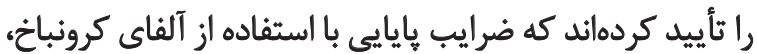

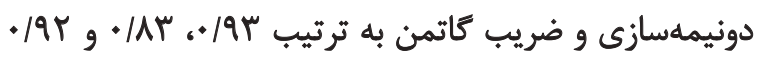

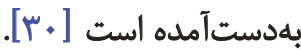

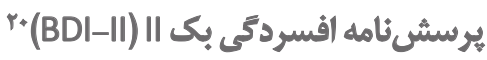

اين يرسشنامه نوع بازنغرى شده BDI است. برسشنامه

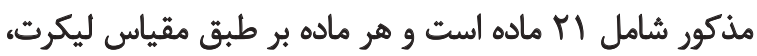

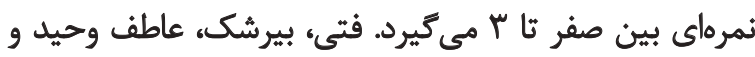

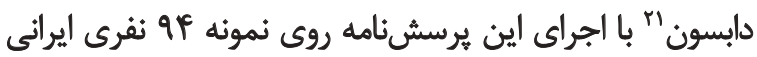

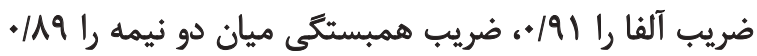

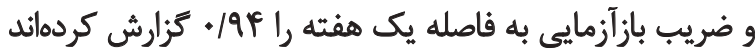

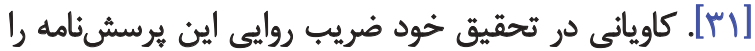

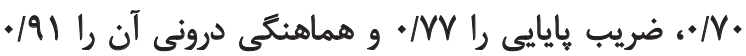

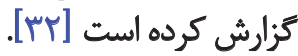

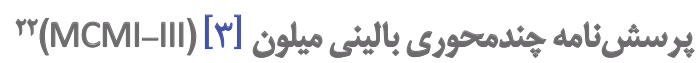

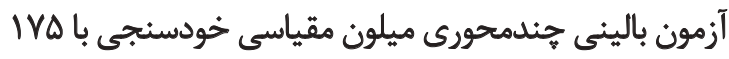

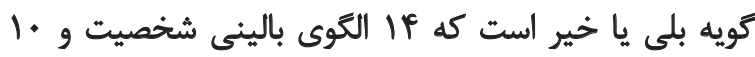

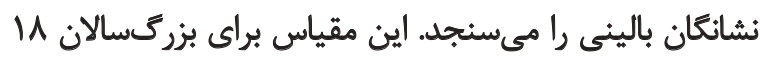

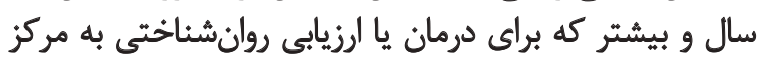

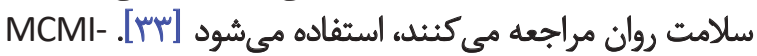

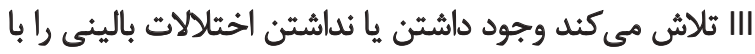

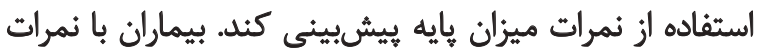

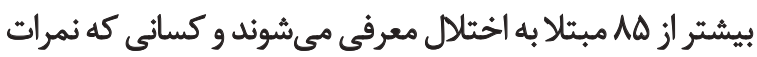

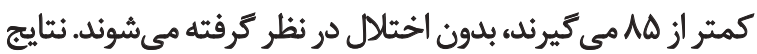

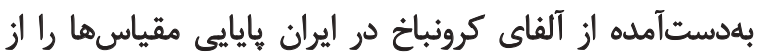

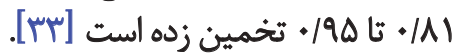

20. Beck Depression Inventory-II

21. Fata, Birashk, Atef-Vahid, Dobson

22. Millon Clinical Multiaxial Inventory-III

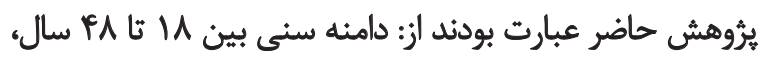

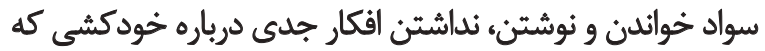

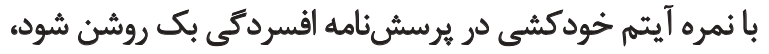

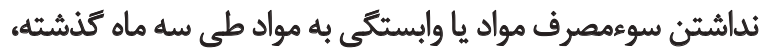

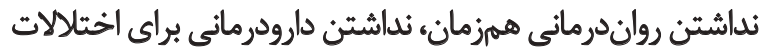

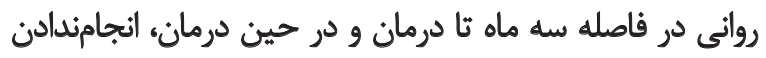

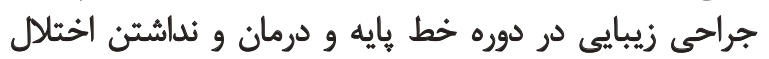

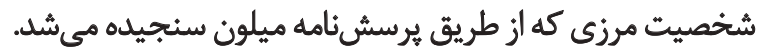
در اين يُؤشش از راهنماى درمان شناختىرفتارى براى

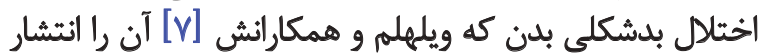

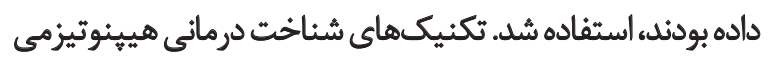

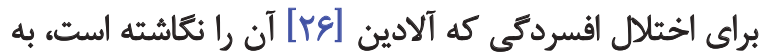

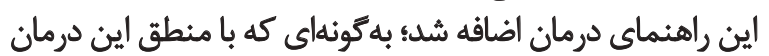

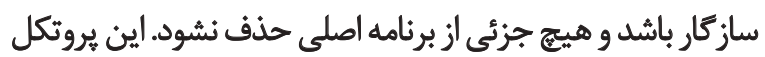

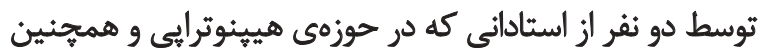

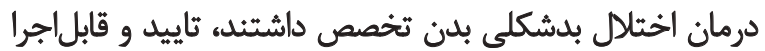

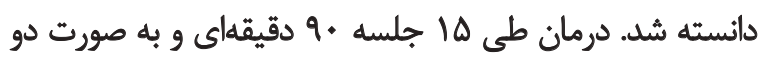

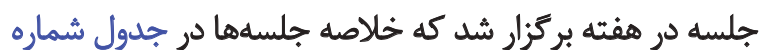
آورده شدهاست.

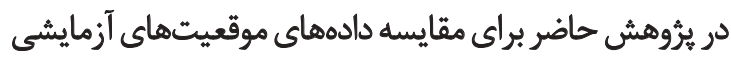

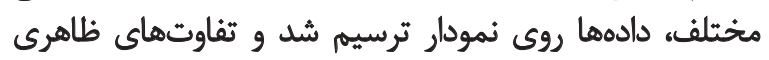

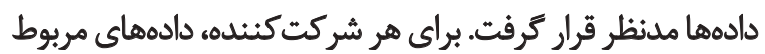

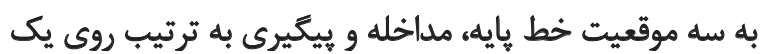

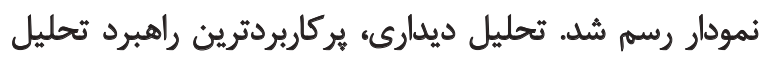

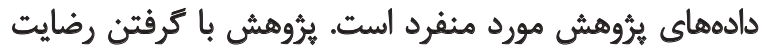

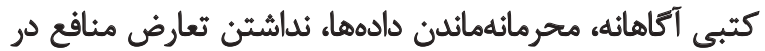

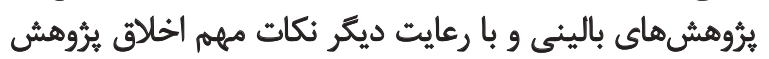

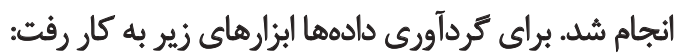

مقياس وسواس فكرى عملى ييل-براون اصلاحشده براى اختلال بدشكلى بدن (BDD-YBOCS)

مقياس Ir إدهاي و نيمه ساختاريافتهاي است كه اجراكنيده

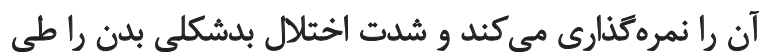

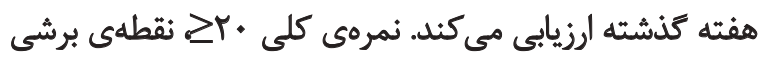

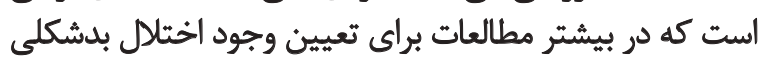

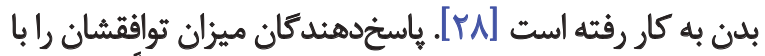

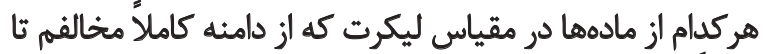

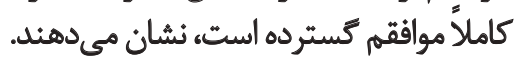

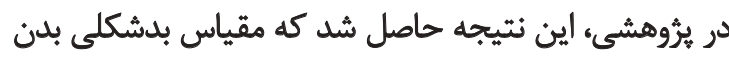

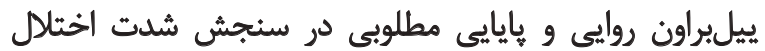

19. Yale-Brown Obsessive Compulsive Scale modified for Body Dysmorphic Disorder 


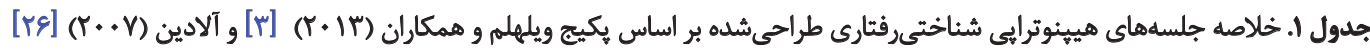

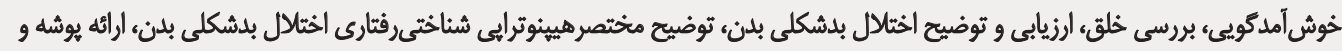

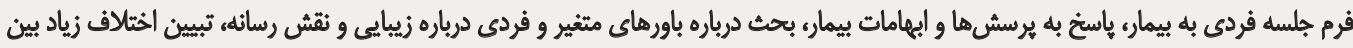
ناهربيرونى و تنانغاره

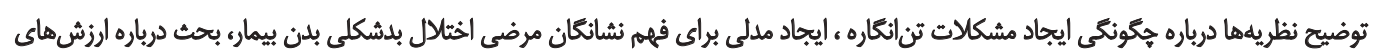

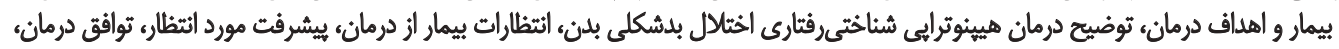

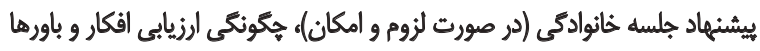

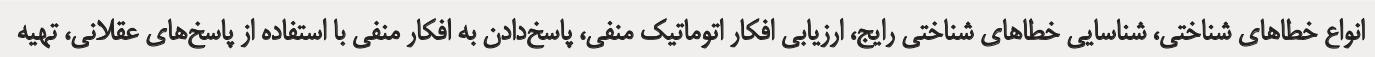

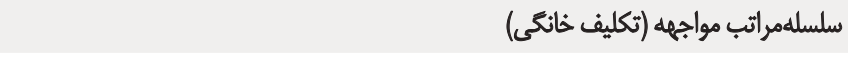

جلسه 1

جلسه

ras

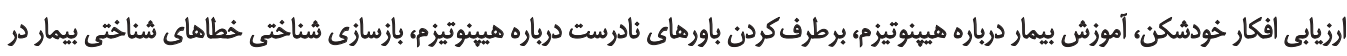

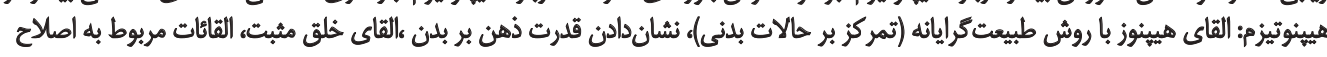

جلسه

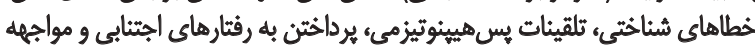

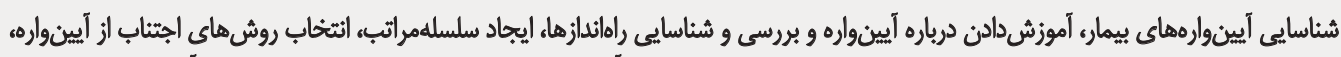

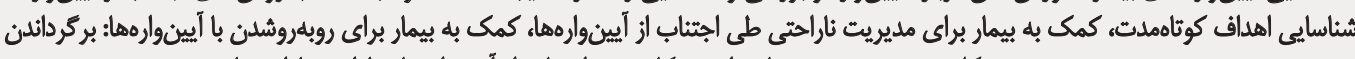

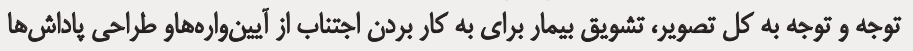

جلسه P

جلسه Q

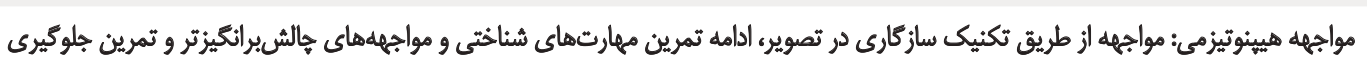
الز ياسخ

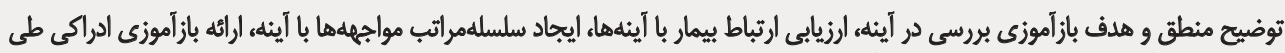

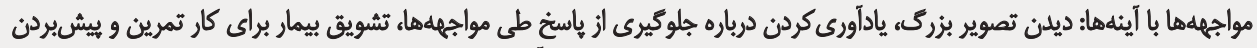

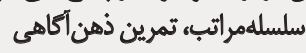

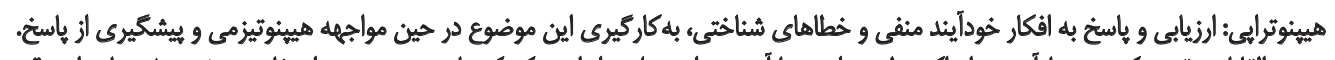

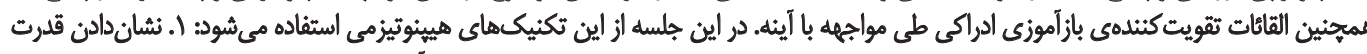

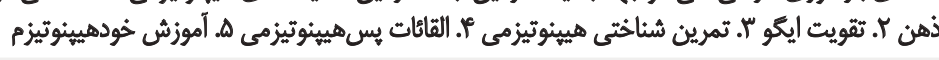

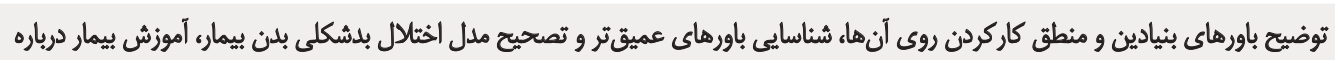

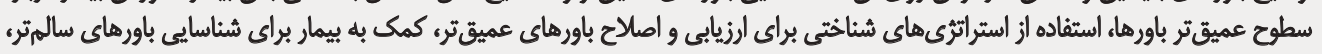

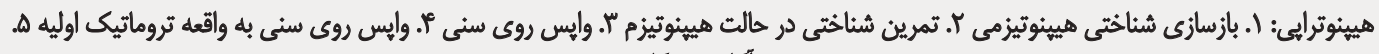

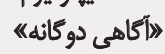

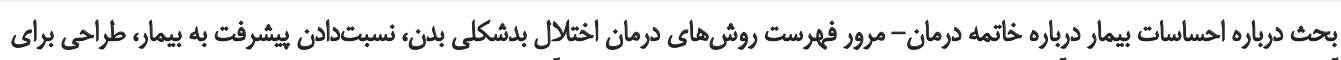

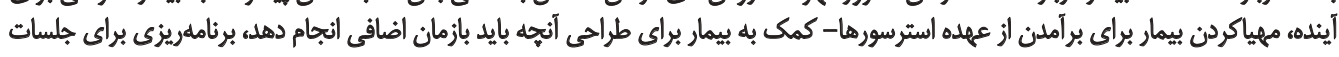

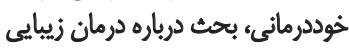

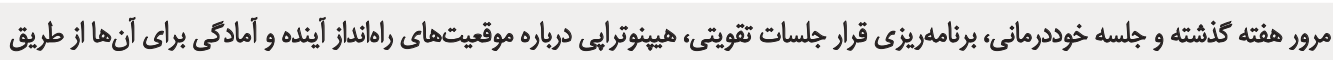

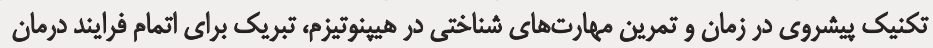

:

جليس

Vجل

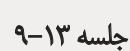

جلسه

جلسه 10

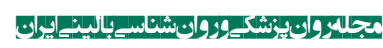

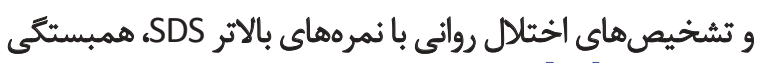

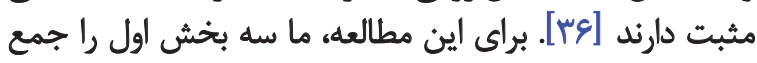

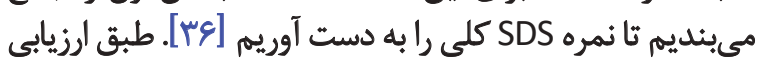

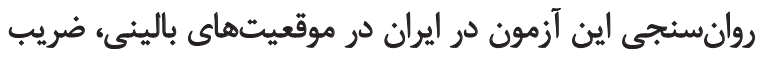

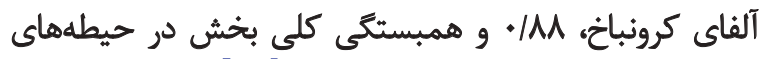

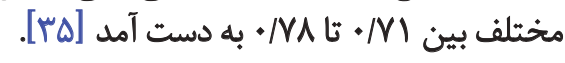

$$
\text { جزئيات بالينى شركت كثثد كَان در بروهش }
$$

بيمار اول

بيمار اول آقايى ·r ساله بود. نكرانى اصلى مراجع مربوط

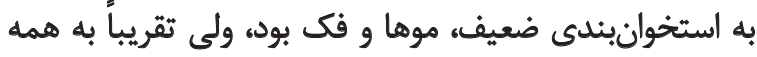

\section{مثياس ناتوانى شيهان (SDS)}

مقياسى خود مزارشى، مربوط به ناتوانى و اختلال عملكرد

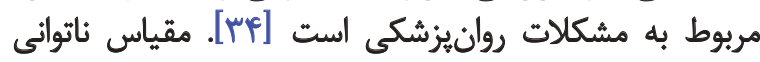

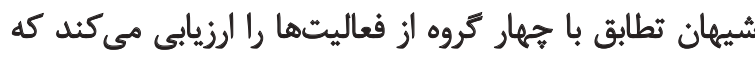

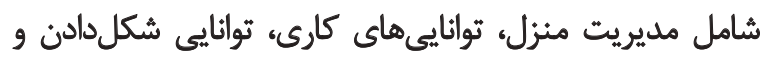

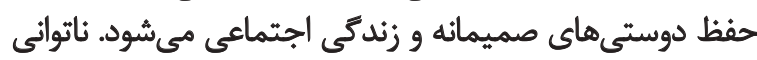

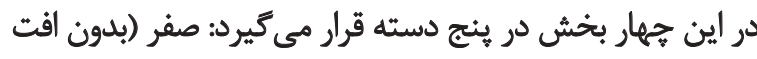

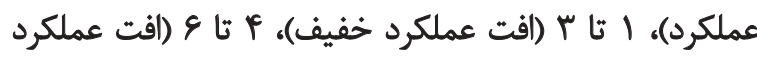

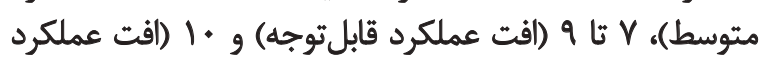

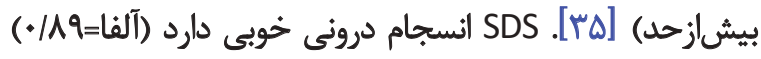

23. Sheehan Disability Scale 


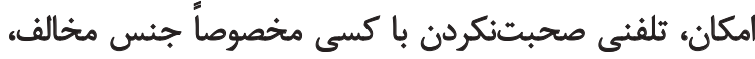

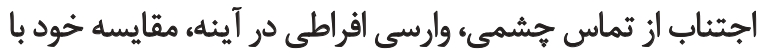

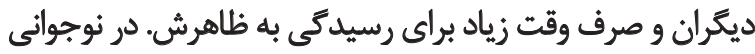

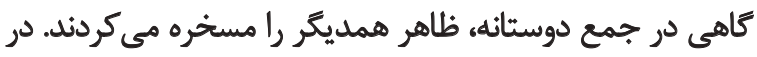

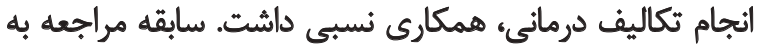

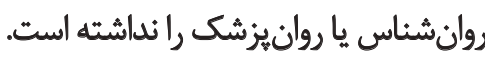

\section{ياقتهها}

ابتدا دادههاي كردآّرى شده از سه موقعيت خط خايايه، مداخله

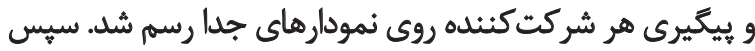

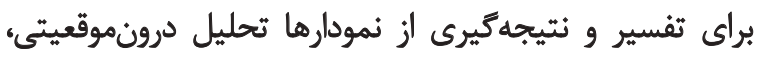

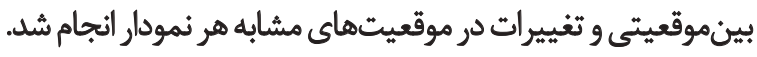

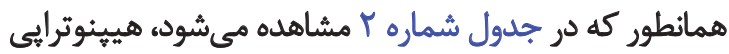

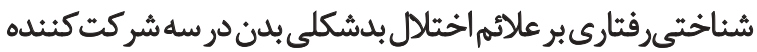

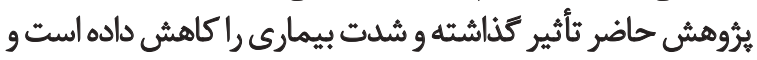

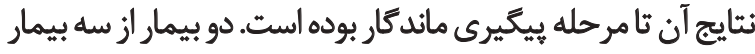

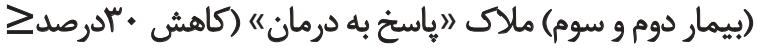

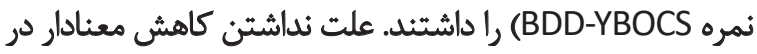

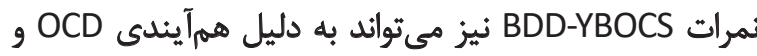

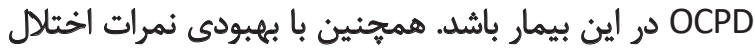
بدشكلى بدن، نمرات ناثوانى و افسردگى نيز دئ در هر سه به بيمار

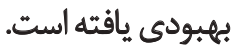

يافتههاى جدول شماره ا به صورت نمودار دادهها در تصاوير

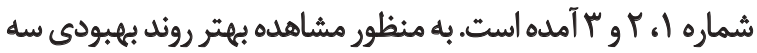

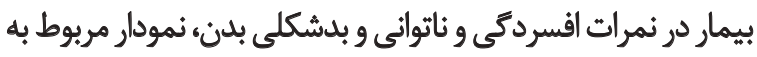

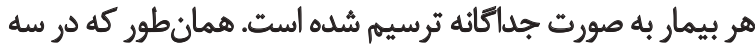

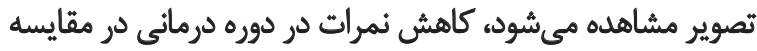

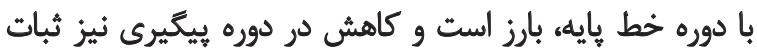

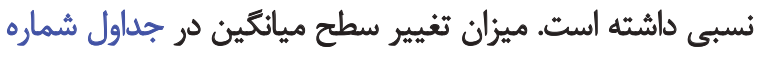

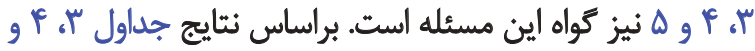

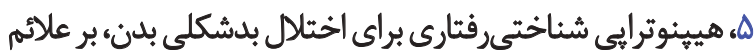

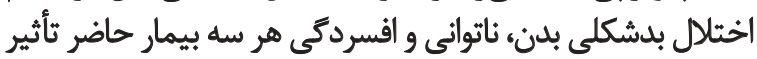
كذاشته است و نتايج آن تا مرحله بيكيرى نيز وانيز ماندكار بوده است.

بحث

مطالعه حاضر بسط و توسعه برنامه الحاقى هيينوترايى به به برائ

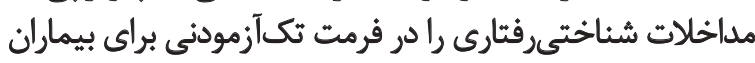

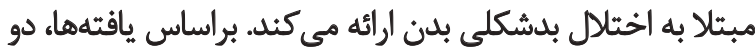

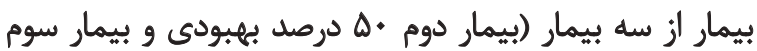

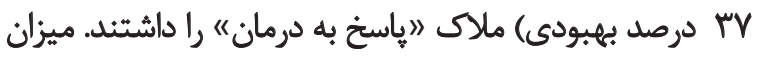

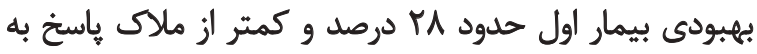

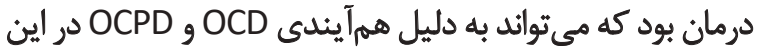

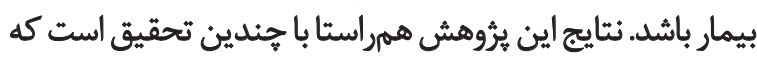

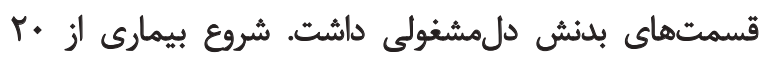

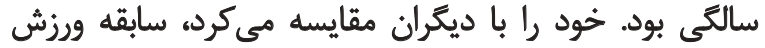

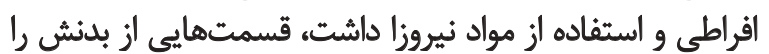

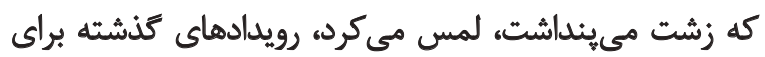

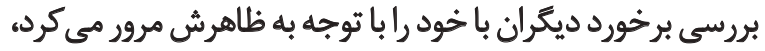

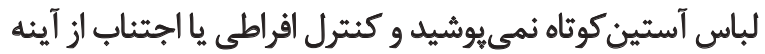

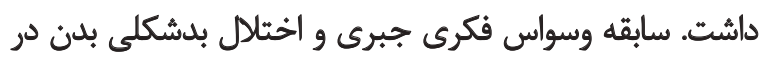

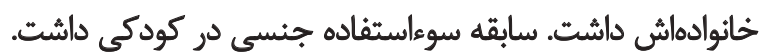

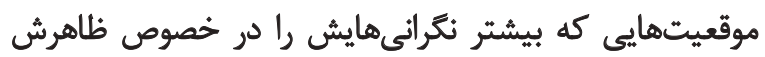

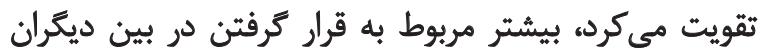

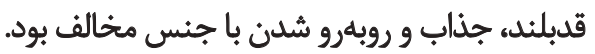

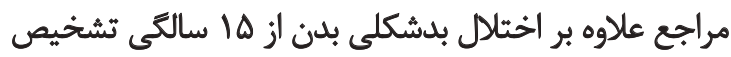

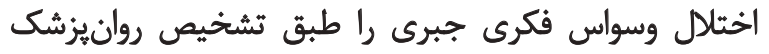

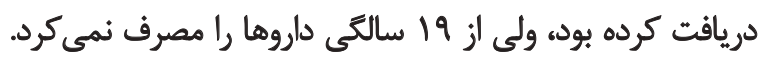

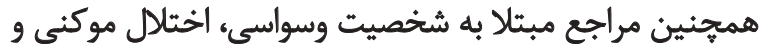

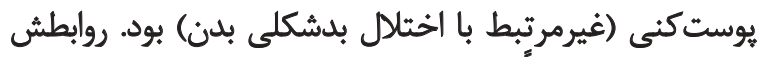

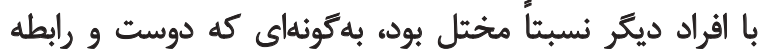

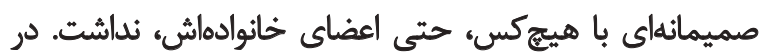
انجام تكاليف همكارى نمى كرد و بهشدت كمال كرا بودي

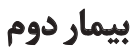
بيمار دوم خانمى الس ساله بود. نتخرانىاش در خصوص ظاهرش

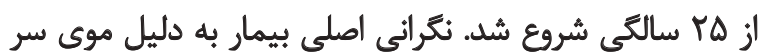

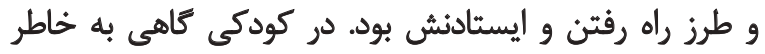

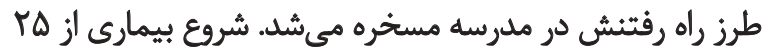

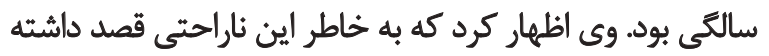

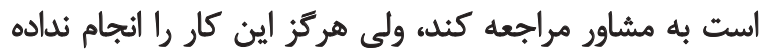

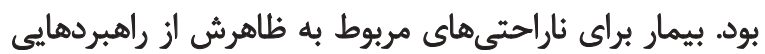

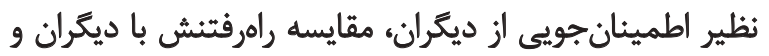

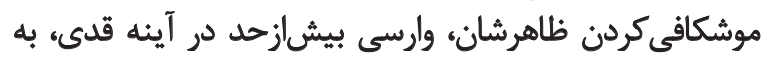

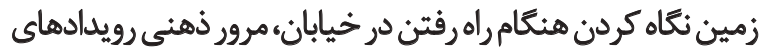

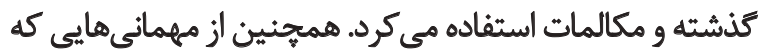

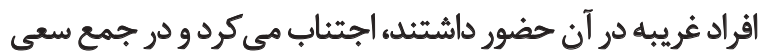

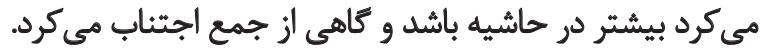

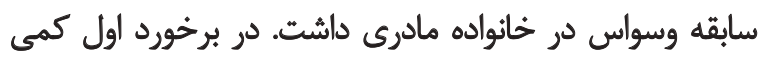

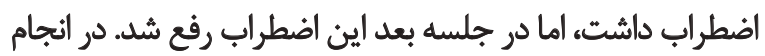
تكاليف همكارى خوبى داشت.

بيمار سوم

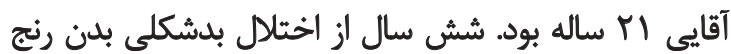

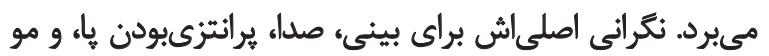

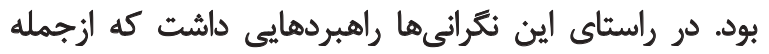

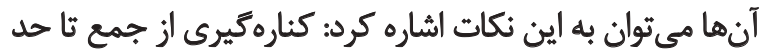




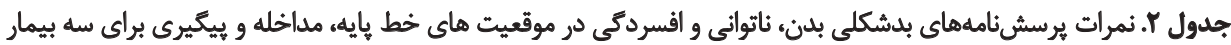

\begin{tabular}{|c|c|c|c|c|c|c|c|c|c|}
\hline افسردكى & ناتوانى & بدشكلى بدن & افسردّىى & ن ناتوانى & بدشكلى بدن & افسردكى & ن اتواثى & بدشكلى بلن & \\
\hline 1. & $r_{*}$ & re & rA & M & rr & re & rf & ro & خط بإيه اول \\
\hline 11 & r. & re & 19 & $M$ & ro & rq & rf & $m$ & خط بايه دوم \\
\hline$\checkmark$ & M & r. & r. & 11 & re & rq & $\pi$ & r & خط يايه سوم \\
\hline 11 & if & r. & $m$ & iv & rf & m & 19 & rA & جلسيه سوم درمان \\
\hline$\checkmark$ & 10 & m & iv & iv & r & rA & in & rV & جلسيه يُنجم درمان \\
\hline$\Lambda$ & 9 & $m$ & $r$ & 10 & $m$ & 19 & $r f$ & re & جلسه هُفتم درمان \\
\hline$\Delta$ & ri & $M$ & 9 & rir & m & 19 & ir & Mr & جلسه نهم درمان \\
\hline r & 9 & iv & 9 & $\vee$ & if & ro & rr & $r \Delta$ & جلسه يازدهم درمان \\
\hline r & 8 & is & 10 & 8 & 18 & if & $M$ & rA & جلسه سيزدهم درمان \\
\hline 1 & $\Delta$ & 10 & 1 & $\Delta$ & ir & 19 & M & mi & جلسه هائزدهم درمان \\
\hline$\varepsilon$ & r & if & 10 & 8 & if & rr & $r$. & ro & ييكيرى جهار ماهل \\
\hline
\end{tabular}

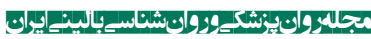

بهبودى در بيكيرى جهارماهل نيز حفظ شد. در يرؤهش حاضر نيز مىبينيم كه بهبودى از نيمه درمان شيب

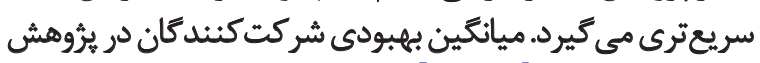

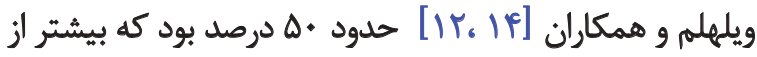

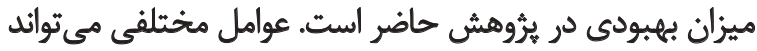

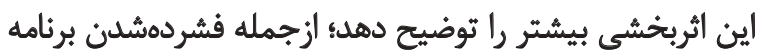

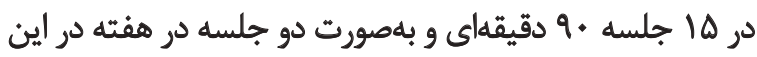

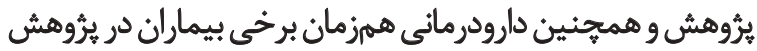

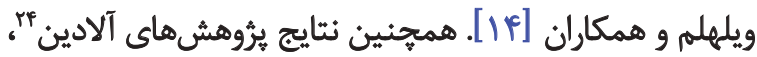

24. Alladin
اثربخشى درمان شناختى فرفتارى بر اختلال بدشكلى بدن را تأييد

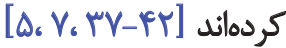

همجينين اين بُروهش همراستا با بثروهش هاي ديكر در زمينه

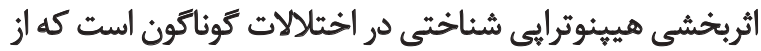

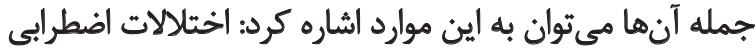

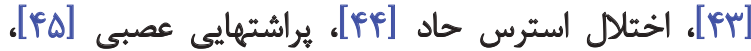

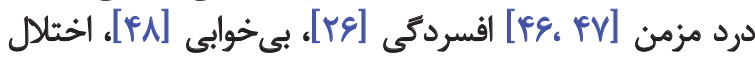

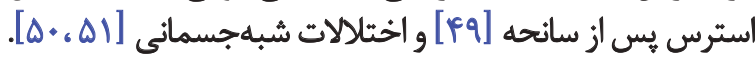

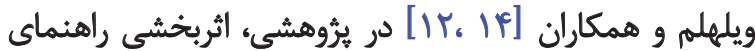

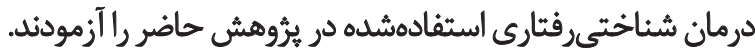

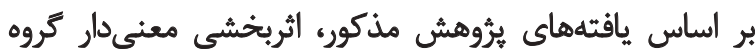

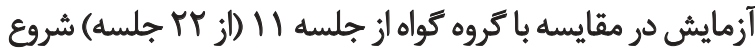

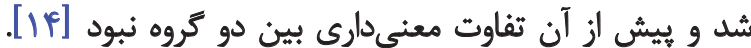

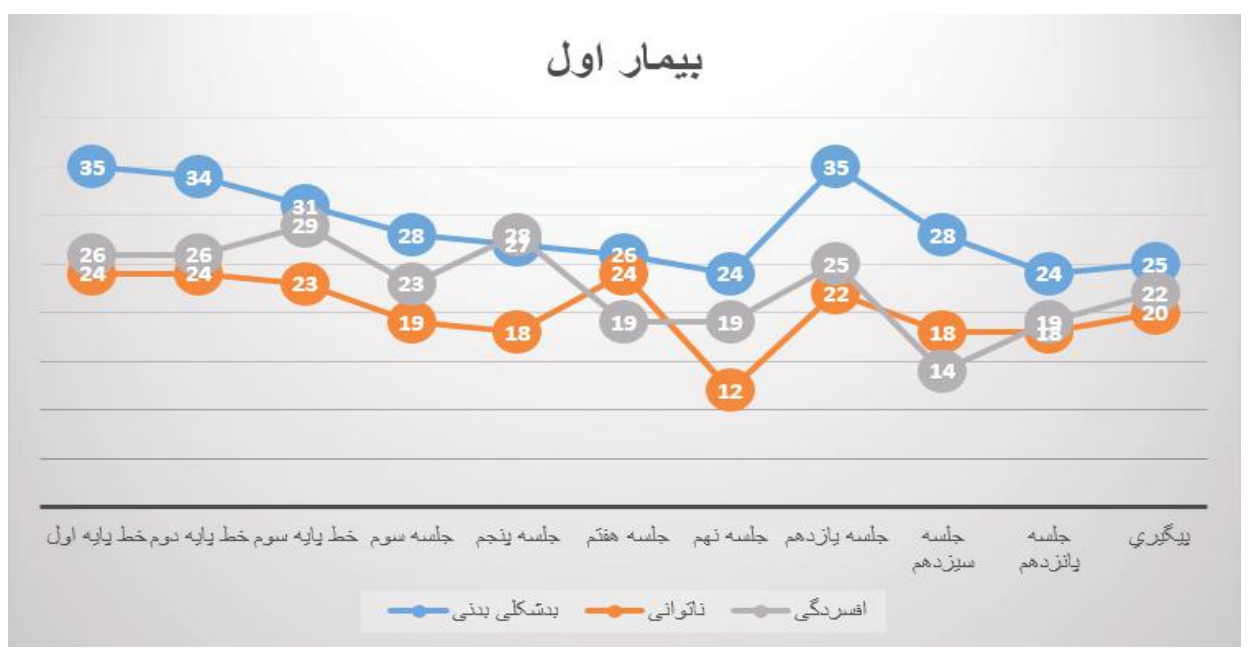




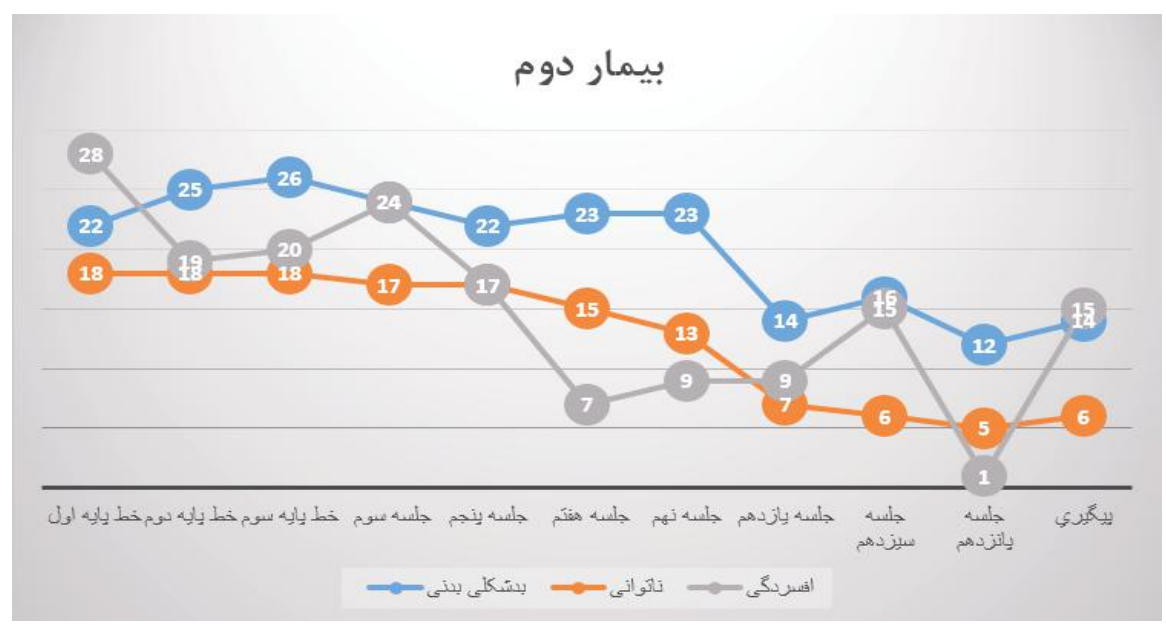

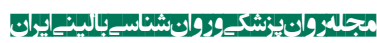

تصوير r. اثربخشى هينوترايى شناختىرفتارى بر بدشكلى بلن، افسردكى و ناتوائى بيمار دوم

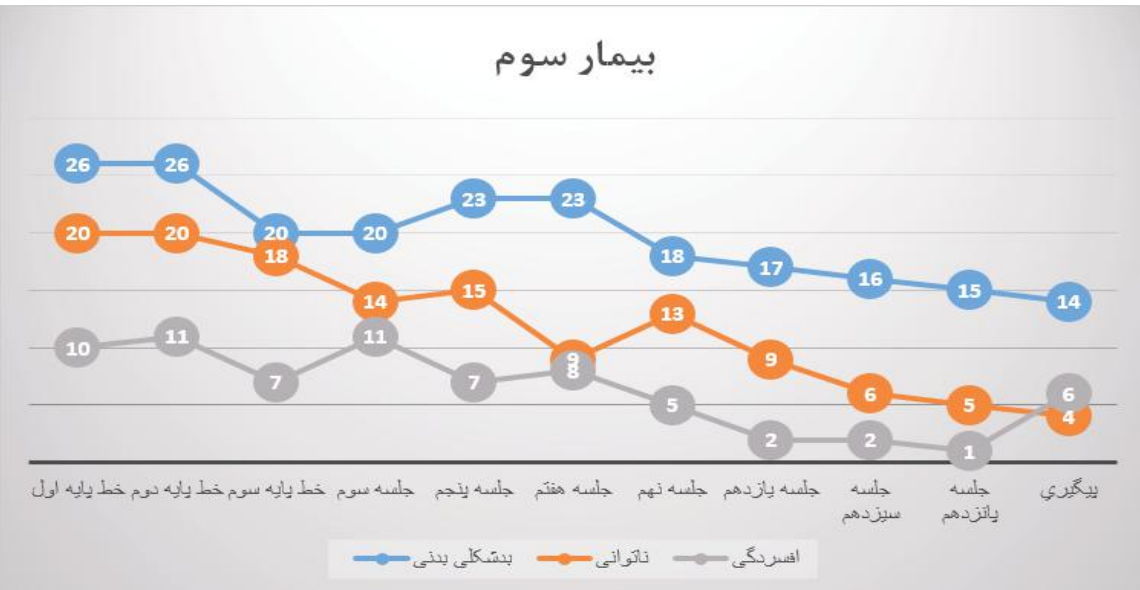

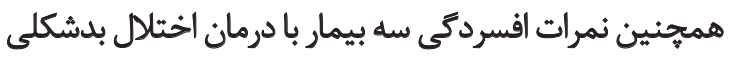

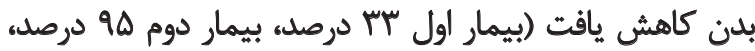

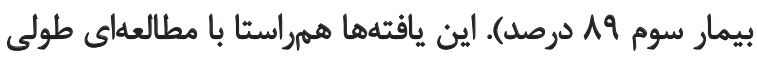

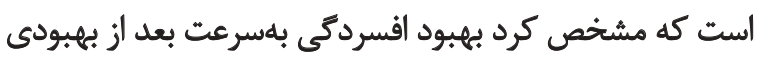

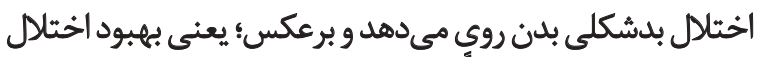

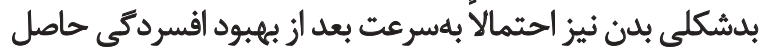

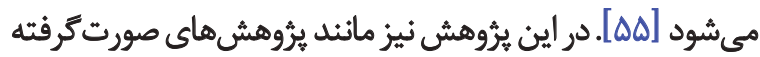

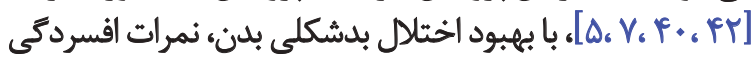

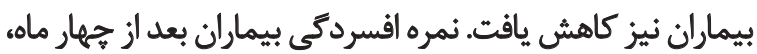

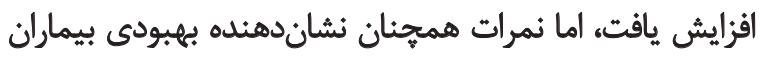

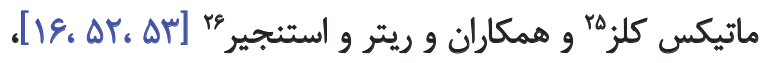

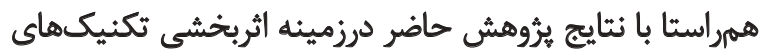

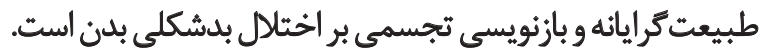

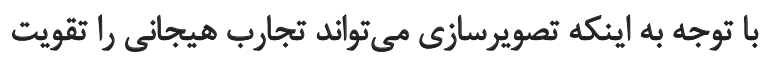

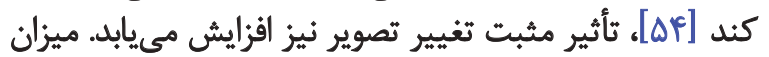

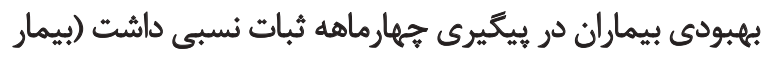

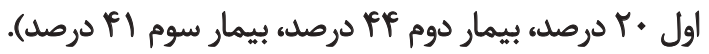

25. Mataix-Cols

26. Ritter \& Stangier

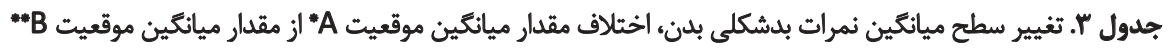

\begin{tabular}{|c|c|c|c|}
\hline ييمار سوم & بيمار دوم & بيمار الول & \\
\hline WNA & $19 / 1 P$ & RV/Pr & Bقدار ميانكين B B \\
\hline rp & $r \varphi / m$ & (m/m & A مقدار مياتكين A \\
\hline ه//10 (بهيود) & ه/19-(بهيود) & (يلهيود) & تغيير سطح ميائكين \\
\hline
\end{tabular}


جدول F. تغيير سطح ميائكين نمرات افسردكى، اختلاف مقدار ميانكين موقعيت A|ز مقدار ميانكين موقعيت B

\begin{tabular}{|c|c|c|c|}
\hline ييمار سوه & بيمار دوم & بيماراول & \\
\hline$\Delta / / F$ & $11 / M$ & M & B مقدار ميانكين \\
\hline $9 / m$ & $\mathrm{TY} / \mathrm{MT}$ & TV & A مقلار مياثكين A \\
\hline 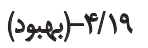 & 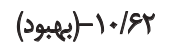 & 8) (بهبود) & تغيير سطع ميائكين \\
\hline
\end{tabular}

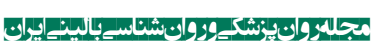

جدول ه. تغيير سطح ميانكين نمرات ثاتوائى، اختلاف مقدار ميائكين موقعيت Aاز مقدار ميائكين موقعيت

\begin{tabular}{|c|c|c|c|}
\hline بيمار سوم & بيمار دوم & بيماراول & \\
\hline $1 . / 14$ & M/AT & $\mid W V$ & Bمثلر ميانكين B مثين \\
\hline $19 / \pi$ & $M$ & Mr/sg & A مقدار ميانكين A \\
\hline 9/19 (بعيود) & 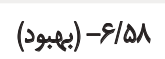 & 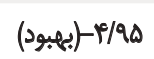 & تغيير سطح ميانكين \\
\hline
\end{tabular}

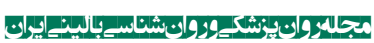

بدشكلى بدن تسهيل مى كند. ضمن اينكه با تفكر واكرايى كه در

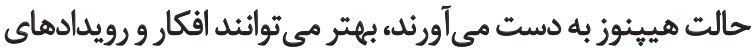

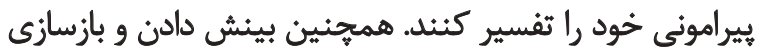

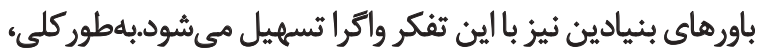

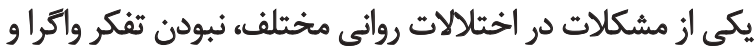

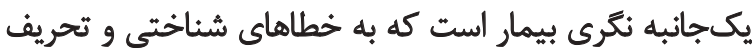

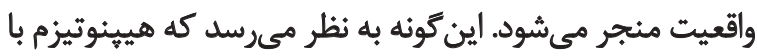

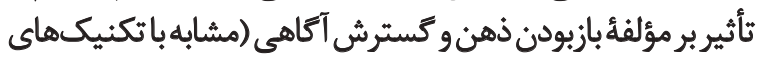

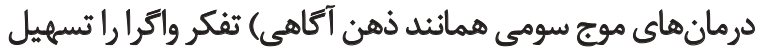
مى كند و در برطرف موحردن اين مشكل، كمك كنينده است.

در يروهش حاضر، شركتكنئدكان بعد از انجام مواجهيه

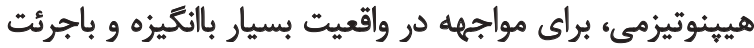

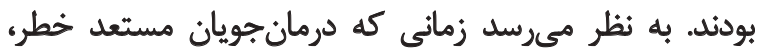

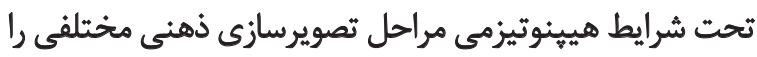

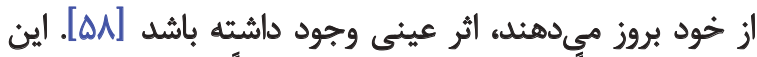

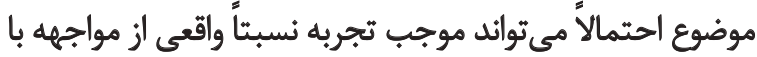

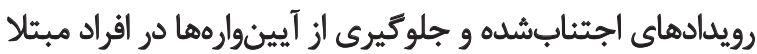

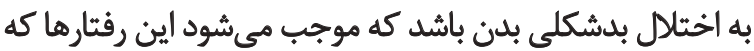

عوامل تداومبخش بيمارى باند، بهتدريج حساسيتز بدايى شودي

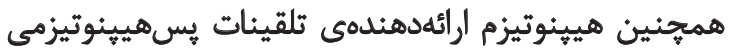

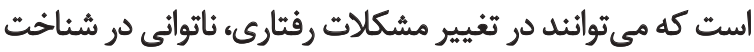

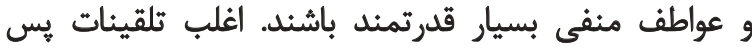

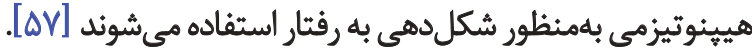

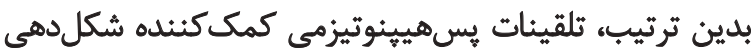

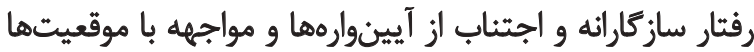

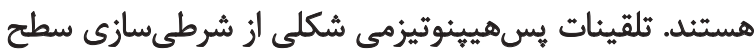

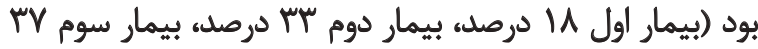

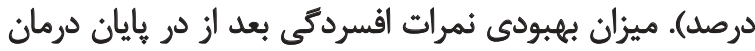

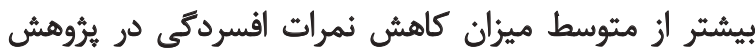

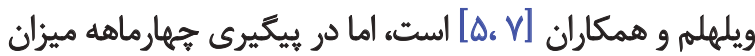

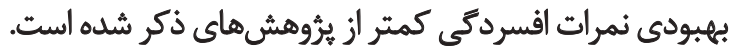
بهبودى اختلال بدشكلى بدن موجب كاهش جشمكير ناتوانى

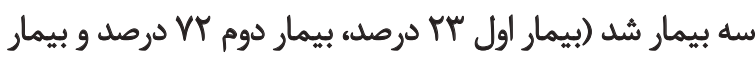

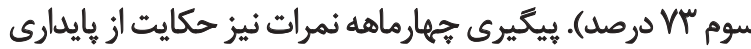

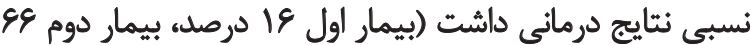

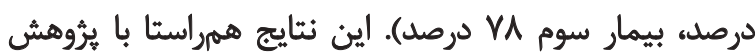
ويلهلم و همكاران [V] [ل است.

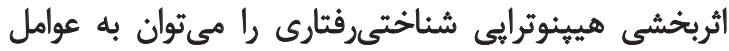

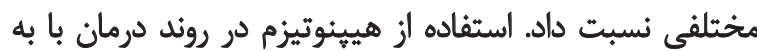

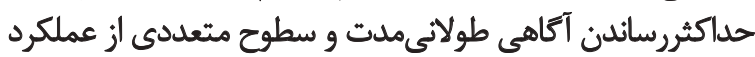

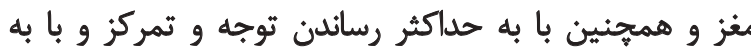

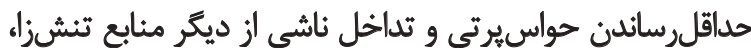

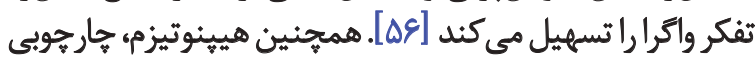

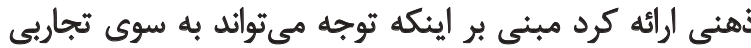

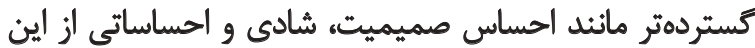

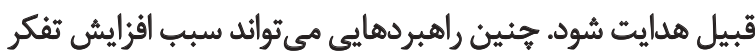

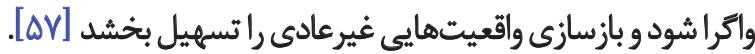
همانطور كه قبلاً ذكر شد، اغلب بيماران مبئلا به اختيلال

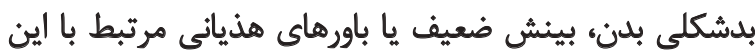

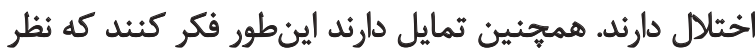

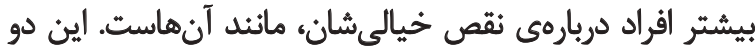

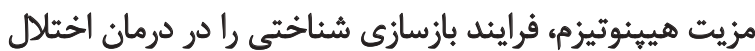


بالاست كه بلهور قابلتوجهى در نقش نيرويى مثبت يا منفى

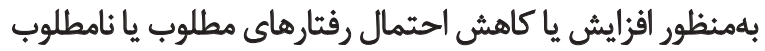

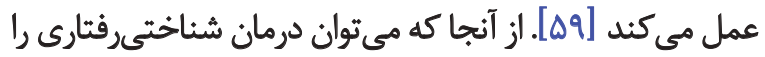

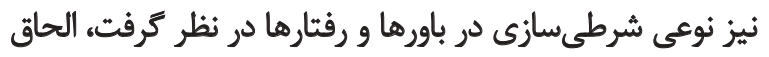

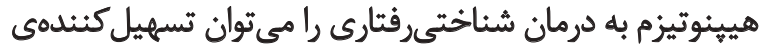

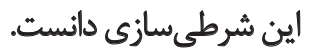

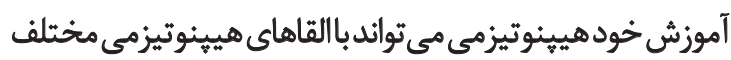

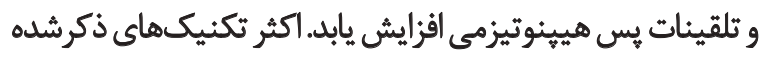

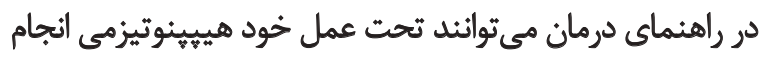

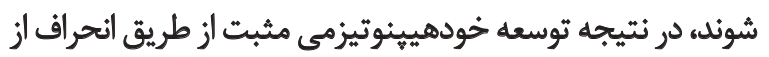

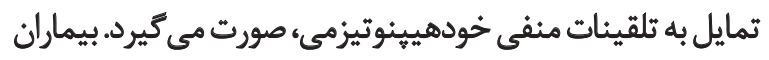

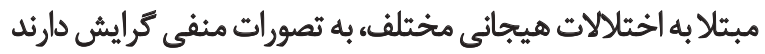

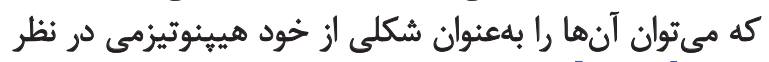

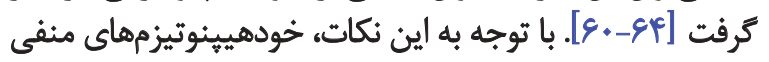

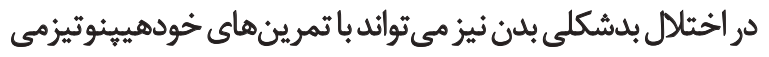

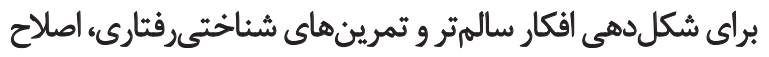

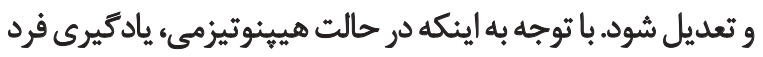

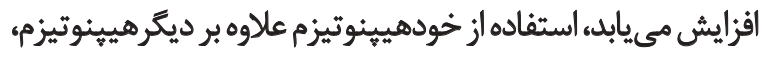

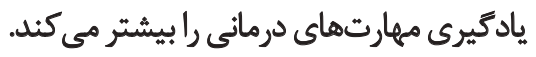

\section{نتيجليَّيرى}

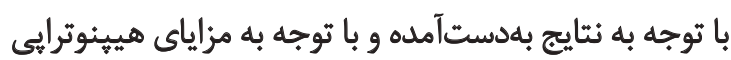

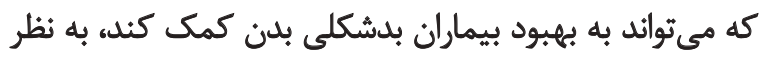

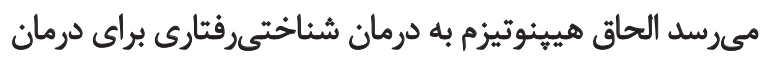

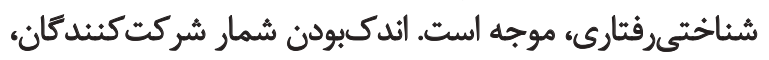

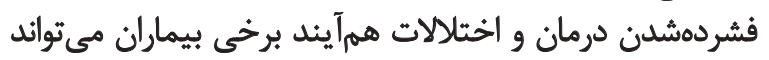

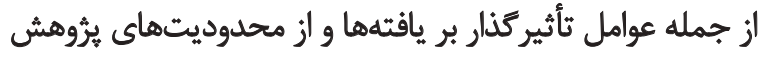

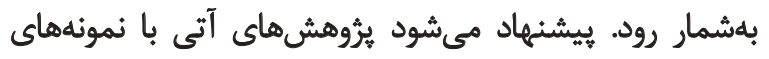

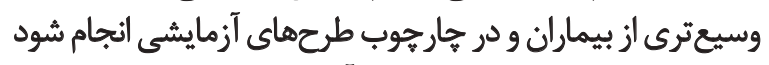

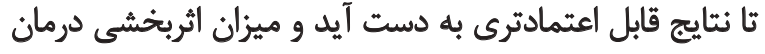

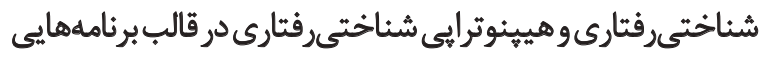

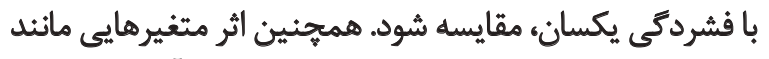

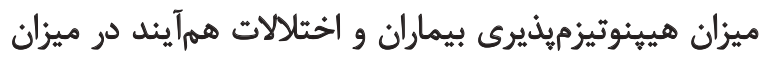

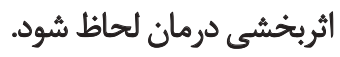

\section{سباسكَّزَارى}

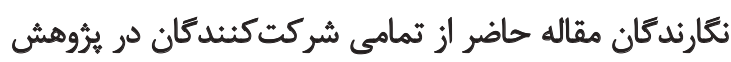

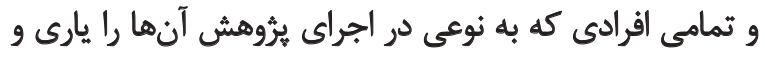

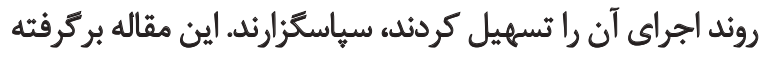

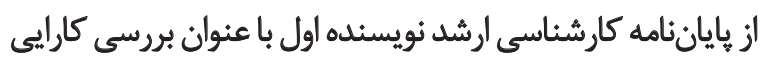

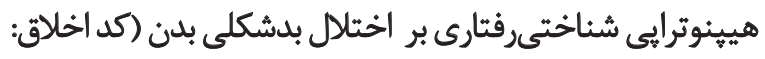

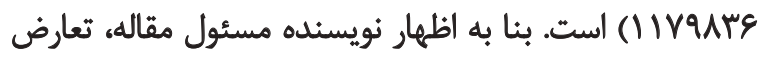
منافع وجود نداشته است. 


\section{References}

[1] American Psychiatric Association. Diagnostic and statistical manual of mental disorders (DSM-5). Arlington: American Psychiatric Association Publishing; 2013.

[2] Phillips KA. Quality of life for patients with Body Dysmorphic Disorder. The Journal of Nervous and Mental Disease. 2000; 188(3):170-5. doi: 10.1097/00005053-200003000-00007

[3] Wilhelm S, Phillips KA, Steketee G. Cognitive-behavioral treatment manual for Body Dysmorphic Disorder. New York: Guilford; 2013.

[4] Ipser JC, Sander C, Stein DJ. Pharmacotherapy and psychotherapy for Body Dysmorphic Disorder. Cochrane Database of Systematic Reviews. 2009; 1. doi: 10.1002/14651858.cd005332

[5] Wilhelm S, Phillips KA, Fama JM, Greenberg JL, Steketee G. Modular cognitive-behavioral therapy for Body Dysmorphic Disorder. Behavior Therapy. 2011; 42(4):624-33. doi: 10.1016/j. beth.2011.02.002

[6] Williams J, Hadjistavropoulos T, Sharpe D. A meta-analysis of psychological and pharmacological treatments for Body Dysmorphic Disorder. Behaviour Research and Therapy. 2006; 44(1):99111. doi: 10.1016/j.brat.2004.12.006

[7] Wilhelm S, Phillips KA, Didie E, Buhlmann U, Greenberg JL, Fama JM, Keshaviah A, Steketee G. Modular cognitive-behavioral therapy for Body Dysmorphic Disorder: A randomized controlled trial. Behavior Therapy. 2014; 45(3):314-27. doi: 10.1016/j. beth.2013.12.007

[8] Prazeres AM, Nascimento AL, Fontenelle LF. Cognitive-behavioral therapy for Body Dysmorphic Disorder: A review of its efficacy. Neuropsychiatric Disease and Treatment. 2013; 9:307. doi: 10.2147/ ndt.s41074

[9] Willson R, Veale D, Freeston M. Imagery rescripting for Body Dysmorphic Disorder: A multiple-baseline single case experimental design. Behavior Therapy. 2016; 47(2):248-61. doi: 10.1016/j. beth.2015.08.006

[10] Veale D. Advances in a cognitive behavioural model of Body Dysmorphic Disorder. Body Image. 2004; 1(1):113-25. doi: 10.1016/ s1740-1445(03)00009-3

[11] Neziroglu F, Khemlani-Patel S, Veale D. Social learning theory and cognitive behavioral models of Body Dysmorphic Disorder. Body Image. 2008; 5(1):28-38. doi: 10.1016/j.bodyim.2008.01.002

[12] Veale D, Neziroglu F. Body Dysmorphic Disorder: A treatment manual. Hoboken, New Jersey: John Wiley \& Sons; 2010.

[13] Araoz DL. The new hypnosis. New York: Brunner-Mazel; 1985.

[14] Golden WL, Dowd ET, Friedberg F. Hypnotherapy: A modern approach. Oxford: Pergamon Press; 1987.

[15] Alladin A, Amundson J. Cognitive hypnotherapy as a transdiagnostic protocol for emotional disorders. International Journal of Clinical and Experimental Hypnosis. 2016; 64(2):147-66. doi: $10.1080 / 00207144.2016 .1131585$

[16] Alladin A. Cognitive hypnotherapy: A new vision and strategy for research and practice. American Journal of Clinical Hypnosis. 2012; 54(4):249-62. doi: 10.1080/00029157.2012.654528
[17] Alladin A. Healing the wounded self: Combining hypnotherapy with ego state therapy. American Journal of Clinical Hypnosis. 2013; 56(1):3-22. doi: 10.1080/00029157.2013.796282

[18] Alladin A. The wounded self: New approach to understanding and treating anxiety disorders. American Journal of Clinical Hypnosis. 2014; 56(4):368-88. doi: 10.1080/00029157.2014.880045

[19] Brown DP, Fromm E. Hypnotherapy and hypnoanalysis. Abingdon: Routledge; 1986

[20] Ewin DM, Eimer BN. Ideomotor signals for rapid hypnoanalysis: A How-to-manual. Sangamon County, Illinois: Charles C Thomas Publisher; 2006

[21] Watkins JG, Barabasz A. Advanced hypnotherapy: Hypnodynamic techniques. Abingdon: Routledge; 2007.

[22] Watkins JG. The affect bridge: A hypnoanalytic technique. International Journal of Clinical and Experimental Hypnosis. 1971; 19(1):21-7. doi: 10.1080/00207147108407148

[23] Yapko MD. Trancework: An introduction to the practice of clinical hypnosis. Abingdon: Routledge; 2012

[24] Barabasz AF, Watkins JG. Hypnotherapeutic techniques. Abingdon: Routledge; 2005.

[25] Hammond DC. Hypnotic induction \& suggestion. Bloomingdale, Illinois: American Society of Clinical Hypnosis; 1998.

[26] Alladin A, Alibhai A. Cognitive hypnotherapy for depression: an empirical investigation. International Journal of Clinical and Experimental Hypnosis. 2007; 55(2):147-66. doi: 10.1080/00207140601177897

[27] Kirsch I, Montgomery G, Sapirstein G. Hypnosis as an adjunct to cognitive-behavioral psychotherapy: A meta-analysis.Consulting and Clinical Psychology. 1995; 63(2):214-20. doi: 10.1037//0022006x.63.2.214

[28] Phillips KA. An open-label study of escitalopram in Body Dysmorphic Disorder. International Clinical Psychopharmacology. 2006; 21(3):177-9. doi: 10.1097/01.yic.0000194378.65460.ef

[29] Phillips KA, Hart AS, Menard W. Psychometric evaluation of the Yale-Brown Obsessive-Compulsive Scale Modified for Body Dysmorphic Disorder (BDD-YBOCS). Journal of Obsessive-Compulsive and Related Disorders. 2014; 3(3):205-8. doi: 10.1016/j. jocrd.2014.04.004

[30] Rabeie M. Khoramdel K. Kalantari M. Molavi H. [Factor structure, reliability and validity of Yale-Brown obsessive-compulsive scale modification of Body Dysmorphic Disorder among students (Persian)]. Iranian Journal of Psychiatry and Clinical Psychology. 2009, 2010; 15(4):343-350

[31] Fata L, Birashk B, Atef-Vahid MK, Dobson KS. [Meaning assignment structures/ schemas, emotional states and cognitive processing of emotional information: Comparision of two conceptual frameworks (Persian)]. Iranian Journal of Psychiatry and Clinical Psychology. 2005; 11(3):312-326.

[32] Kaviani H. [Validity and reliability of hospital anxiety and depression scale (HADS), general health questionnaire (GHQ-28), Mood Adjectives Checklist, \& BDI in clinical population in comparison with healthy group; Research report (Persian)]. Tehran: Tehran University of Medical Sciences; 2008. 
[33] Sharifi AA, Molavi H, Namdari K. [The validity of MCMI-III (Millon) scales (Persian)]. Knowledge \& Research in Applied Psychology. 2005; 9(34):27-38.

[34] Sheehan DV, Harnett-Sheehan K, Raj BA. The measurement of disability. International Clinical Psychopharmacology. 1996; 11(3):89-95. doi: 10.1097/00004850-199606003-00015

[35] Amin-Esmaeili M, Motevalian A, Rahimi-Movaghar A, Hajebi A, Hefazi M, Radgoodarzi R, Sharifi V. The Translation and Psychometric Assessment of the Persian Version of the Sheehan Disability Scale. Iranian Journal of Psychiatry. 2014; 9(3):125-32. PMCID: PMC4277800

[36] Rush Jr J, First MB, Blacker D. Handbook of psychiatric measures. Arlington: American Psychiatric Publishing; 2007.

[37] Neziroglu F, McKay D, Todaro J, Yaryura-Tobias JA. Effect of cognitive behavior therapy on persons with Body Dysmorphic Disorder and comorbid axis II diagnoses. Behavior Therapy. 1997; 27(1):67-77. doi: 10.1016/s0005-7894(96)80036-0

[38] Veale D, Gournay K, Dryden W, Boocock A, Shah F, Willson R, Walburn J. Body Dysmorphic Disorder: a cognitive behavioural model and pilot randomised controlled trial. Behaviour Research and Therapy. 1996; 34(9):717-29. doi: 10.1016/0005-7967(96)00025-3

[39] McKay D, Todaro J, Neziroglu F, Campisi T, Moritz EK, YaryuraTobias JA. Body Dysmorphic Disorder: A preliminary evaluation of treatment and maintenance using exposure with response prevention. Behaviour Research and Therapy. 1997; 35(1):67-70. doi: 10.1016/s0005-7967(96)00082-4

[40] Geremia GM, Neziroglu F. Cognitive therapy in the treatment of Body Dysmorphic Disorder. Clinical Psychology \& Psychotherapy. 2001; 8(4):243-51. doi: 10.1002/cpp.284

[41] Rosen JC, Reiter J, Orosan P. Cognitive-behavioral body image therapy for Body Dysmorphic Disorder. Journal of Consulting and Clinical Psychology. 1995; 63(2):263-69. doi: 10.1037//0022006x.63.2.263

[42] Wilhelm S, Otto MW, Lohr B, Deckersbach T. Cognitive behavior group therapy for Body Dysmorphic Disorder: a case series. Behaviour Research and Therapy. 1999; 37(1):71-5. doi: 10.1016/ s0005-7967(98)00109-0

[43] Golden WL. Cognitive hypnotherapy for anxiety disorders. American Journal of Clinical Hypnosis. 2012; 54(4):263-74. doi: 10.1080/00029157.2011.650333

[44] Bryant RA, Moulds ML, Guthrie RM, Nixon RDV. The additive benefit of hypnosis and cognitive-behavioral therapy in treating acute stress disorder. Journal of Consulting and Clinical Psychology. 2005; 73(2):334-40. doi: 10.1037/0022-006x.73.2.334

[45] Barabasz M. Cognitive hypnotherapy with bulimia. American Journal of Clinical Hypnosis. 2012; 54(4):353-64. doi: 10.1080/00029157.2012.658122

[46] Elkins G, Johnson A, Fisher W. Cognitive hypnotherapy for pain management. American Journal of Clinical Hypnosis. 2012; 54(4):294-310. doi: 10.1080/00029157.2011.654284

[47] Jensen MP. Hypnosis for chronic pain management. New York: Oxford University Press; 2013.

[48] Graci GM, Hardie JC. Evidenced-based hypnotherapy for the management of sleep disorders. International Journal of Clinical and Experimental Hypnosis. 2007; 55(3):288-302. doi: 10.1080/00207140701338662
[49] Lynn SJ, Cardeña E. Hypnosis and the treatment of posttraumatic conditions: An evidence-based approach. International Journal of Clinical and Experimental Hypnosis. 2007; 55(2):167-88. doi: 10.1080/00207140601177905

[50] Alladin A. Cognitive hypnotherapy: An integrated approach to the treatment of emotional disorders. Hoboken, New Jersey: John Wiley \& Sons; 2008.

[51] Moene FC, Spinhoven P, Hoogduin KA, Dyck RV. A randomized controlled clinical trial of a hypnosis-based treatment for patients with conversion disorder, motor type. International Journal of Clinical and Experimental Hypnosis. 2003; 51(1):29-50. doi: 10.1076/iceh.51.1.29.14067

[52] Mataix-Cols D, Fernández de la Cruz L, Isomura K, Anson M, Turner C, Monzani B, et al. A pilot randomized controlled trial of cognitive-behavioral therapy for adolescents with Body Dysmorphic Disorder. Journal of the American Academy of Child \& Adolescent Psychiatry. 2015; 54(11):895-904. doi: 10.1016/j. jaac.2015.08.011

[53] Ritter V, Stangier U. Seeing in the Mind's eye: Imagery rescripting for patients with Body Dysmorphic Disorder. A single case series. Journal of Behavior Therapy and Experimental Psychiatry. 2016; 50:187-95. doi: 10.1016/j.jbtep.2015.07.007

[54] Holmes EA, Mathews A. Mental imagery in emotion and emotional disorders. Clinical Psychology Review. 2010; 30(3):349-62. doi: 10.1016/j.cpr.2010.01.001

[55] Phillips KA, Stout RL. Associations in the longitudinal course of Body Dysmorphic Disorder with major depression, obsessivecompulsive disorder, and social phobia. Journal of Psychiatric Research. 2006; 40(4):360-9. doi: 10.1016/j.jpsychires.2005.10.001

[56] Tosi DJ, Baisden BS. Cognitive-experiential therapy and hypnosis. Clinical hypnosis: A multidisciplinary approach. In: Alladin A, editor. Cognitive Hypnotherapy: An Integrated Approach to the Treatment of Emotional Disorders. Hoboken, New Jersey: John Wiley \& Sons; 2008.

[57] Alladin A. Cognitive hypnotherapy: An integrated approach to the treatment of emotional disorders. Hoboken, New Jersey: John Wiley \& Sons; 2008.

[58] Lazarus, Arnold A. A multimodal framework for clinical hypnosis. In: Alladin A, Editor. Cognitive hypnotherapy: An integrated approach to the treatment of emotional disorders. Hoboken, New Jersey: John Wiley \& Sons; 2008.

[59] Barrios AA. Posthypnotic suggestion as higher-order conditioning: A methodological and experimental analysis. International Journal of Clinical and Experimental Hypnosis. 1973; 21(1):32-50. doi: $10.1080 / 00207147308409303$

[60] Alladin A. Cognitive hypnotherapy with depression. Journal of Cognitive Psychotherapy. 1994; 8(4):275-88.

[61] Chapman RA. The clinical use of hypnosis in cognitive behavior therapy: a practitioner's casebook. Berlin: Springer; 2005.

[62] Alladin A. Special issue: Evidence-based practice in clinical hypnosis - Part II. International Journal of Experimental and Clinical Hypnosis. 2007; 55(3):251-371.

[63] Araoz DL. Negative self-hypnosis. Journal of Contemporary Psychotherapy. 1981; 12(1):45-52. doi: 10.1007/bf00946233

[64] Araoz DL. The new hypnosis. New York: Brunner-Mazel; 1977. 